\title{
Vibration Analysis of Thin Plate Structures Subjected to a Moving Force Using Frequency-Domain Spectral Element Method
}

\author{
Taehyun Kim (iD) and Usik Lee 1 (D) \\ Department of Mechanical Engineering, Inha University, Inha-ro 100, Nam-gu, Incheon 22212, Republic of Korea \\ Correspondence should be addressed to Usik Lee; ulee@inha.ac.kr
}

Received 21 June 2018; Accepted 14 August 2018; Published 24 September 2018

Academic Editor: Francesco Franco

Copyright (c) 2018 Taehyun Kim and Usik Lee. This is an open access article distributed under the Creative Commons Attribution License, which permits unrestricted use, distribution, and reproduction in any medium, provided the original work is properly cited.

\begin{abstract}
A frequency-domain spectral element method (SEM) is proposed for the vibration analysis of thin plate structures subjected to a moving point force. The thin plate structures may consist of multiple rectangular thin plates with arbitrary boundary conditions that form multispan thin plate structures, such as bridges. The time-domain point force moving on a thin rectangular plate with arbitrary trajectory is transformed into a series of stationary point forces in the frequency domain. The vibration responses induced by the moving point force are then obtained by superposing all vibration responses excited by each stationary point force. For the vibration response of a specific stationary point force, the plate subjected to the specific stationary point force is represented by four spectral finite plate elements, which were developed in the authors' previous work. The SEM-based vibration analysis technique is first presented for single-span thin plate structures and then extended to the multispan thin plate structures. The high accuracy and computational efficiency of the proposed SEM-based vibration analysis technique are verified by comparison with other well-known solution methods, such as the exact theory, integral transform method, finite element method, and the commercial finite element analysis package ANSYS.
\end{abstract}

\section{Introduction}

In the structural engineering field, moving load problems, which deal with vibrations of structures under moving forces or masses, have been important research subjects over the last few decades. Typical examples of moving load problems are overhead cranes, ballistic systems, cableways, railway tracks, magnetic disks, building slabs, and bridges. These structures under moving loads have been modeled either as one-dimensional (1D) or two-dimensional (2D) structures. For the reliable design and maintenance of such structures, it is essential to predict the vibration characteristics of the structures accurately, using an efficient analysis method.

For the vibration analysis of $1 \mathrm{D}$ and $2 \mathrm{D}$ moving load problems, numerous analysis methods have been proposed in the literature. These include the modal analysis method (mode superposition method or eigenfunction expansion method) [1-4], integral transform method (ITM) [5-7], Galerkin method $[8,9]$, differential quadrature method $[10,11]$, finite difference method $[12,13]$, finite element method (FEM) [14-18], dynamic stiffness method [19, 20], and frequency-domain spectral element method (SEM) [21-25].

To investigate the vibration characteristics of complex structures subjected to moving loads, the FEM has been the most widely used. The shape functions used in the FEM are independent of the vibration frequencies of a structure. Thus, the FEM generally requires a very fine discretization of the structure to acquire reliable solutions, especially in the high-frequency range. This may result in a significant increase in computational cost.

Thus, the frequency-domain SEM based on the fast Fourier transform (FFT) theory $[26,27]$ has been suggested as an alternative to the FEM. The spectral element matrix (or dynamic stiffness matrix) used in the SEM is formulated from the frequency-dependent dynamic shape functions derived from free-wave solutions that satisfy the governing equations of motion in the frequency domain. Thus, the SEM can provide highly accurate solutions by representing a uniform structure member as a single finite element, regardless of its size, and thus significantly reduce the computational cost compared to the FEM. 
Despite the outstanding features of the SEM, it has been applied to very few moving load problems, for instance, in [21-24] for 1D structures and in [25] for 2D structures. For 1D structures, Azizi et al. [21] seem to be the first to have applied the SEM to continuous beams and bridges under a moving point force. They discretized a uniform beam structure into many finite elements (more than two) and represented a moving point force using effective nodal forces and moments acting on each of the nodes of a finite beam element, as commonly done using the FEM. Later, Sarvestan et al. [22] adopted the SEM technique developed by Azizi et al. [21] for the vibration analysis of a cracked Bernoulli-Euler beam subjected to a moving point force. Recently, for the vibration analysis of a Timoshenko beam subjected to a moving point force, Song et al. [23] presented a new SEM technique. They represented the time-domain moving point force by a series of stationary point forces in the frequency domain. Thereafter, by using the superposition principle, they obtained the time-domain vibration responses to the moving point force by summing and taking the inverse Fourier transform of all individual frequencydomain vibration responses to each stationary point force. For the vibration responses excited by a specific stationary point force, they used the so-called two-element method, where the Timoshenko beam was discretized into two finite elements so that the stationary point force was located at their joint. To avoid the structural discretization required for the two-element method used in Song et al. [23], Kim and Lee [28] introduced a spectral element modeling technique, called the modified one-element method, where exact solutions can be obtained by simply adding some correction terms, formulated in analytical forms, to the solutions by the one-element model. Kim and Lee [24] applied the modified one-element method to the vibration analysis of multispan beams subjected to a moving point force. For the 2D structures, Shirmohammadi et al. [25] seem to be the first to have applied the SEM to the vibration analysis of rectangular plates subjected to a moving point force. They assumed that the point force was moving at a constant speed and the trajectory of the moving point force was parallel to the one edge of a rectangular plate. For the SEM analysis, they used the spectral finite strip element model developed for a Levytype plate, and, in the frequency domain, they transformed the moving point force into the modal form by using the separation of variable method. Thus, the application of their SEM technique is limited to the Levy-type plates. Through a thorough literature survey, we found that there have been no studies yet in which the SEM has been applied to moving load-induced vibrations of a thin plate structure with arbitrary boundary conditions.

For the SEM-based vibration analysis of a thin plate structure with arbitrary boundary conditions, the formulation of a generic type of spectral plate element model that can be applied to any boundary conditions has been required. Recently, Park et al. $[29,30]$ developed such a generic type of spectral plate element model for the thin isotropic and composite rectangular plates with arbitrary boundary conditions by using the boundary splitting method [31] and the spectral super element method [32]. However, to the authors' best knowledge, there are no reports in the literature on the application of the generic type of spectral plate element model to the vibration analysis of a thin plate structure with arbitrary boundary conditions, subjected to a moving point force with arbitrary trajectory.

Thus, we propose a new SEM-based vibration analysis method for isotropic single-span or multispan thin plate structures with arbitrary boundary conditions, subjected to a moving point force by using the generic type of spectral plate element model developed by Park et al. [29]. By extending the frequency-domain representation of a moving point force moving on a beam structure [23], a point force that moves along an arbitrary trajectory on a thin plate is transformed into the frequency domain as a series of stationary point forces distributed along the trajectory of this moving point force. The vibration responses induced by the moving point force are then obtained by superposing all individual vibration responses excited by each stationary point force. An individual vibration response excited by a specific stationary point force is obtained by representing the rectangular plate span on which the specific stationary point force is located using the four-element model, where the specific stationary point force is located at the junction of four spectral plate elements. All the other plate spans, without the stationary point force, are represented by the two-element model. The high accuracy of the solution and computational efficiency of the newly proposed SEM-based vibration analysis method are verified by comparison with other solution techniques, such as the exact theory, ITM, FEM, and the well-known commercial finite element analysis package ANSYS. In addition, the effects of the boundary conditions, moving speed, and the trajectory of a moving force on the vibration responses of the single-span square plates and three-span rectangular plates are numerically investigated.

\section{Problem Statement}

Consider a thin isotropic uniform rectangular plate (simply, plate) having arbitrary boundary conditions, subjected to a moving point force with a constant magnitude $P$, as shown in Figure 1 . The length and width of the plate are denoted by $l_{x}$ and $l_{y}$, respectively. The red dotted line represents the arbitrary trajectory of the moving point force, and the current positions of the moving point force with respect to the $x$ - and $y$-coordinates are denoted by $s_{x}(t)$ and $s_{y}(t)$, respectively.

Using the classical plate theory [33], the governing equation of the plate subjected to a moving point force $P$ can be written as

$$
\begin{aligned}
& D\left(\frac{\partial^{4} w}{\partial x^{4}}+2 \frac{\partial^{4} w}{\partial x^{2} \partial y^{2}}+\frac{\partial^{4} w}{\partial x^{4}}\right)+\rho h \frac{\partial^{2} w}{\partial t^{2}} \\
& \quad=f(x, y, t) \\
& \quad=P \delta\left(x-s_{x}(t)\right) \delta\left(y-s_{y}(t)\right)
\end{aligned}
$$

where $w(x, y, t)$ is the transverse displacement; $D=E h^{3} /[12$ $\left.\left(1-v^{2}\right)\right]$ is the bending stiffness of the plate, where $E$ is Young's modulus, $v$ is Poisson's ratio, and $h$ is the plate 


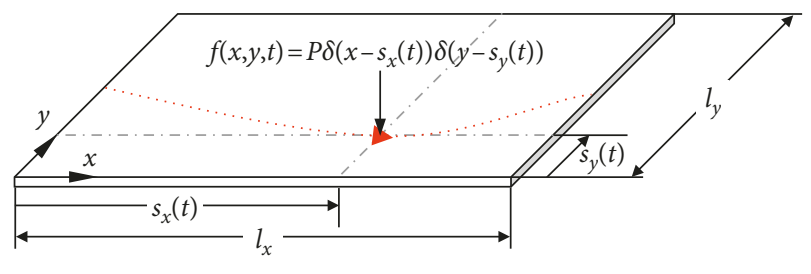

Figure 1: A thin rectangular plate subjected to a moving point force $P$, where the red arrow indicates current position of the moving point force.

thickness; $\rho$ is the mass density; and $f(x, y, t)=P \delta(x-$ $\left.s_{x}(t)\right) \delta\left(y-s_{y}(t)\right)$ represents a moving point force of magnitude $P$, where $\delta(x)$ and $\delta(y)$ are delta functions [34].

\section{Vibration Analysis of a Plate Subjected to a Moving Point Force}

3.1. Frequency-Domain Representation of a Moving Point Force. For the frequency-domain vibration analysis of the plate subjected to a $2 \mathrm{D}$ moving point force $f(x, y, t)$, it is required to transform the $2 \mathrm{D}$ moving point force $f(x, y, t)$ into the frequency domain. Similar to the frequency-domain representation of a $1 \mathrm{D}$ moving point force considered in the previous study [23], the $2 \mathrm{D}$ moving point force $f(x, y, t)$ can be transformed into the frequency domain, using the discrete Fourier transform theory (DFT) [35], as follows:

$$
\begin{array}{r}
\bar{f}_{m}\left(x, y, \omega_{m}\right)=\sum_{n=0}^{N-1} f_{n}(x, y) e^{-i \omega_{m} t_{n}}, \\
(m=0,1,2, \ldots, N-1),
\end{array}
$$

where

$$
\begin{gathered}
f_{n}(x, y)=P \delta\left(x-s_{x(n)}\right) \delta\left(y-s_{y(n)}\right), \\
s_{x(n)}=s_{x}\left(t_{n}\right), s_{x(n)}=s_{y}\left(t_{n}\right),
\end{gathered}
$$

where $i=\sqrt{-1}$ is the imaginary unit. In (2), $\bar{f}_{m}\left(x, y, \omega_{m}\right)$ are spectral components of $f(x, y, t), N$ is the number of spectral components up to the Nyquist frequency [35], and $t_{n}=n \Delta t$, where $\Delta t=T / N$ is the time increment and $T$ is the time window.

Using the delta function, a continuous function $g(x, y)$ can be approximated in terms of its sampling as follows [36]:

$$
g(x, y)=\sum_{k=0}^{K} \sum_{j=0}^{K} g\left(s_{x(j)}, s_{y(k)}\right) \delta\left(x-s_{x(j)}\right) \delta\left(y-s_{y(k)}\right) .
$$

By using (4), (2) can be written as follows:

$$
\begin{aligned}
\bar{f}_{m}\left(x, y, \omega_{m}\right)= & \sum_{k=0}^{K} \sum_{j=0}^{K}\left\{\sum_{n=0}^{N-1} P \delta\left(s_{x(j)}-s_{x(n)}\right)\right. \\
& \left.\cdot \delta\left(s_{y(k)}-s_{y(n)}\right) e^{-i \omega_{m} t_{n}}\right\} \\
& \times \delta\left(x-s_{x(j)}\right) \delta\left(y-s_{y(k)}\right) .
\end{aligned}
$$

Because the delta functions $\delta\left(s_{x(j)}-s_{x(n)}\right)$ and $\delta\left(s_{y(k)}-\right.$ $\left.s_{y(n)}\right)$ differ from zero when $n=j$ and $n=k$, respectively, (5) can be rewritten as

$$
\bar{f}_{m}\left(x, y, \omega_{m}\right)=\sum_{k=0}^{K} P e^{-i \omega_{m} t_{k}} \delta\left(x-s_{x(k)}\right) \delta\left(y-s_{y(k)}\right),
$$

where $K \leq N-1$. By omitting the subscripts $m$ for brevity, (6) can be written in a simple form as follows:

$$
\bar{f}(x, y, \omega)=\sum_{k=0}^{K} F_{k} \delta\left(x-s_{x(k)}\right) \delta\left(y-s_{y(k)}\right),
$$

where

$$
F_{k}=P e^{-i \omega t_{k}}
$$

As shown in (7), the frequency-domain representation of a moving point force $P$ is given by a series of stationary point forces $F_{k}(k=0,1,2, \ldots, K)$ defined by (8). As illustrated in Figure 2, all stationary point forces are distributed along the trajectory of the moving point force. The total number of stationary point forces on the plate is denoted by $K+1 . K$ is dependent on the sizes of $T$ and $T_{\mathrm{A}}$, where $T_{\mathrm{A}}$ is the traveling time required for a point force to move from one end to the other end of its trajectory [23]: (1) $K=N-1$ when $T \leq T_{\mathrm{A}}$; (2) $K<N-1$ when $T>T_{\mathrm{A}}$. In Figure 2, the position of a stationary point force $F_{k}$ is denoted by $\left(s_{x(k)}, s_{y(k)}\right)$, where

$$
\begin{aligned}
& 0 \leq s_{x(k)} \leq l_{x}, \\
& 0 \leq s_{y(k)} \leq l_{y} .
\end{aligned}
$$

\subsection{Spectral Element Modeling for a Plate Subjected to a Stationary Point Force}

3.2.1. Spectral Element Model for a Rectangular Finite Plate Element. Consider a rectangular finite plate element as shown in Figure 3. The rectangular finite plate element has dimensions $l_{\zeta}$ and $l_{\eta}$ with respect to the local coordinates $\zeta$ and $\eta$, respectively. In [29], the spectral element model for a rectangular finite plate element was developed based on the Kantorovich-based finite strip element method where the rectangular finite plate element was divided into $N_{\zeta}$ finite strip elements in the direction of coordinate $\zeta$ and $N_{\eta}$ finite strip elements in the direction of coordinate $\eta$. The spectral element model developed in [29] can be cited in the following form:

$$
\mathbf{S}_{(e)}\left(\omega ; N_{\zeta}, N_{\eta}, l_{\zeta}, l_{\eta}\right) \mathbf{d}_{(e)}=\mathbf{f}_{c(e)}+\mathbf{f}_{d(e)} .
$$

where $S_{(e)}(\omega)$ is the $8\left(N_{\zeta}+N_{\eta}\right)$-by- $8\left(N_{\zeta}+N_{\eta}\right)$ symmetric spectral element matrix (or dynamic stiffness matrix) and $\mathbf{d}_{(e)}$ represents the spectral nodal degrees of freedom (simply, nodal DOFs) vector defined by

$$
\mathbf{d}_{(e)}=\left\{\mathbf{d}^{\mathrm{C}(1) T} \mathbf{d}^{\mathrm{LT}} \mathbf{d}^{\mathrm{C}(2) T} \mathbf{d}^{\mathrm{C}(3) T} \mathbf{d}^{\mathrm{RT}} \mathbf{d}^{\mathrm{C}(4) T} \mathbf{d}^{\mathrm{B} T} \mathbf{d}^{\mathrm{U} T}\right\}^{\mathrm{T}} .
$$




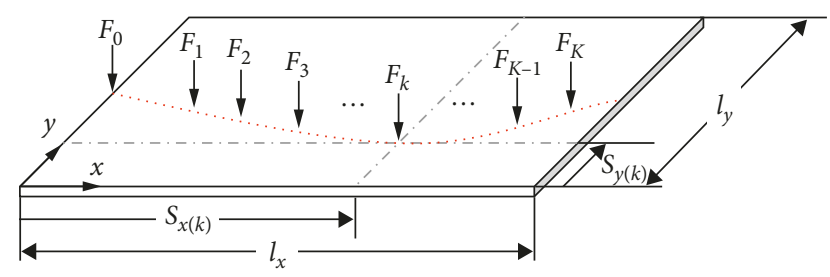

FIGURE 2: Frequency-domain representation of a moving point force $P$ as a series of stationary point forces $F_{k}(k=0,1,2, \ldots, K)$.

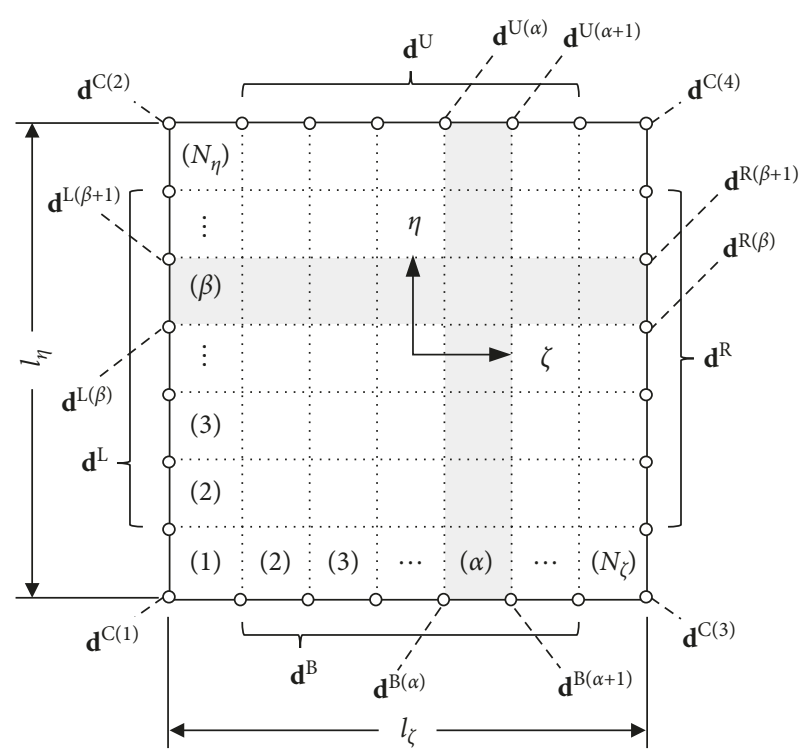

FIGURE 3: Spectral nodal DOFs for a rectangular finite plate element, where $\mathrm{O}=$ nodes.

where $\mathbf{d}^{\mathrm{L}}$ and $\mathbf{d}^{\mathrm{R}}$ represent $4\left(N_{\eta}-1\right)$ nodal DOFs at the nodes on the left and right edges of the finite plate element, respectively, excluding the nodal DOFs at four corner nodes; $\mathbf{d}^{\mathrm{B}}$ and $\mathbf{d}^{\mathrm{U}}$ represent $4\left(N_{\zeta}-1\right)$ nodal DOFs at the nodes on the bottom and upper edges, respectively, excluding the nodal DOFs at four corner nodes; and $\mathbf{d}^{\mathrm{C}(r)}$ $(r=1,2,3,4)$ represents four nodal DOFs at each corner node, as shown in Figure 3. In (10), the quantities after the symbol ";" denote the parameters appeared in the spectral element matrix $\boldsymbol{S}_{(e)}(\omega)$ from [29]. In (10), $\mathbf{f}_{c(e)}$ represents the nodal reaction forces and moments on the boundary edges and $\mathbf{f}_{d(e)}$ represents the nodal forces and moments contributed by external loadings. In (11), the superscripts $\mathrm{T}$ denote the transpose of the vector quantities. The nodal DOFs at a node on the left, right, bottom, and upper edges, and at a corner node, are defined by

$$
\begin{aligned}
\mathbf{d}^{\mathrm{L}(\beta)}= & \left\{\bar{w}^{\mathrm{L}(\beta)} \bar{\theta}_{\zeta}^{\mathrm{L}(\beta)} \bar{\theta}_{\eta}^{\mathrm{L}(\beta)} \bar{\theta}_{\zeta \eta}^{\mathrm{L}(\beta)}\right\}_{\mathrm{T}}, \\
\mathbf{d}^{\mathrm{R}(\beta)}= & \left\{\bar{w}^{\mathrm{R}(\beta)} \bar{\theta}_{\zeta}^{\mathrm{R}(\beta)} \bar{\theta}_{\eta}^{\mathrm{R}(\beta)} \bar{\theta}_{\zeta \eta}^{\mathrm{R}(\beta)}\right\}_{\mathrm{T}}, \\
& \left(\beta=2,3,4, \ldots, N_{\eta}\right) \\
\mathbf{d}^{\mathrm{B}(\alpha)}= & \left\{\bar{w}^{\mathrm{B}(\alpha)} \bar{\theta}_{\zeta}^{\mathrm{B}(\alpha)} \bar{\theta}_{\eta}^{\mathrm{B}(\alpha)} \bar{\theta}_{\zeta \eta}^{\mathrm{B}(\alpha)}\right\}_{\mathrm{T}}, \\
\mathbf{d}^{\mathrm{U}(\alpha)}= & \left\{\bar{w}^{\mathrm{U}(\alpha)} \bar{\theta}_{\zeta}^{\mathrm{U}(\alpha)} \bar{\theta}_{\eta}^{\mathrm{U}(\alpha)} \bar{\theta}_{\zeta \eta}^{\mathrm{U}(\alpha)}\right\}_{\mathrm{T}}, \\
& \left(\alpha=2,3,4, \ldots, N_{\zeta}\right) \\
\mathbf{d}^{\mathrm{C}(r)}= & \left\{\bar{w}^{\mathrm{C}(r)} \bar{\theta}_{\zeta}^{\mathrm{C}(r)} \bar{\theta}_{\eta}^{\mathrm{C}(r)} \bar{\theta}_{\zeta \eta}^{\mathrm{C}(r)}\right\}_{\mathrm{T}}, \quad(r=1,2,3,4),
\end{aligned}
$$

where $\bar{w}$ are the nodal transverse displacements, $\bar{\theta} \bar{\zeta}=\partial \bar{w} / \partial \zeta$ and $\bar{\theta}_{\eta}=\partial \bar{w} / \partial \eta$ are the nodal slopes with respect to the coordinate $\zeta$ and the coordinate $\eta$, respectively, and $\overline{\theta_{\zeta \eta}}=$ $\partial^{2} \bar{w} / \partial \zeta \partial \eta$ are the nodal twist angles.

\subsubsection{Four-Element Model for a Rectangular Plate Subjected} to a Stationary Point Force. A rectangular plate subjected to a stationary point force (say, the $k$-th stationary point force, $F_{k}$ ) can be represented by four finite plate elements as shown in Figure 4. The stationary point force $F_{k}$ is placed at the junction of four finite elements and its location is denoted by $\left(s_{x}, s_{y}\right)$.

By assembling all spectral element equations for the four finite plate elements shown in Figure 4, and by using the same assembly process commonly used in the standard FEM, we can obtain an assembled spectral element equation as follows:

$$
\mathbf{S}(\omega) \mathbf{d}=\mathbf{f}_{c}+\mathbf{f}_{d},
$$

where

$$
\mathbf{S}(\omega)=\sum_{e=1}^{4} \mathbf{T}_{(e)}^{\mathrm{T}} \mathbf{S}_{(e)}\left(\omega ; N_{x(e)}, N_{y(e)}, l_{x(e)}, l_{y(e)}\right) \mathbf{T}_{(e)},
$$

and

$$
\begin{aligned}
& \mathbf{d}=\left\{\mathbf{d}_{(1)}^{\mathrm{C}(1) T} \mathbf{d}_{(1)}^{\mathrm{LT} T} \mathbf{d}_{(3)}^{\mathrm{C}(1) T} \mathbf{d}_{(3)}^{\mathrm{LT} T} \mathbf{d}_{(3)}^{\mathrm{C}(2) T} \mathbf{d}_{(1)}^{\mathrm{B} T} \mathbf{d}_{(1)}^{\mathrm{UT} T} \mathbf{d}_{(3)}^{\mathrm{UT} T} \mathbf{d}_{(1)}^{\mathrm{C}(3) T} \mathbf{d}_{(1)}^{\mathrm{RT}} \mathbf{d}_{F}^{T} \mathbf{d}_{(3)}^{\mathrm{R} T} \mathbf{d}_{(3)}^{\mathrm{C}(4) T} \mathbf{d}_{(2)}^{\mathrm{B} T} \mathbf{d}_{(2)}^{\mathrm{UT} T} \mathbf{d}_{(4)}^{\mathrm{UT} T} \mathbf{d}_{(2)}^{\mathrm{C}(3) T} \mathbf{d}_{(2)}^{\mathrm{R} T} \mathbf{d}_{(4)}^{\mathrm{C}(3) T} \mathbf{d}_{(4)}^{\mathrm{R} T} \mathbf{d}_{(4)}^{\mathrm{C}(4) T}\right\}^{\mathrm{T}}, \\
& \mathbf{f}_{c}=\left\{\mathbf{f}_{c(1)}^{C(1) T} \mathbf{f}_{c(1)}^{L T} \mathbf{f}_{c(3)}^{C(1) T} \mathbf{f}_{c(3)}^{L T} \mathbf{f}_{c(3)}^{C(2) T} \mathbf{f}_{c(1)}^{B T} \mathbf{0}^{\mathrm{T}} \mathbf{f}_{c(3)}^{U T} \mathbf{f}_{c(1)}^{C(3) T} \mathbf{0}^{\mathrm{T}} \mathbf{0}^{\mathrm{T}} \mathbf{0}^{\mathrm{T}} \mathbf{f}_{c(3)}^{C(4) T} \mathbf{f}_{c(2)}^{B T} \mathbf{0}^{\mathrm{T}} \mathbf{f}_{c(4)}^{U T} \mathbf{f}_{c(2)}^{C(3) T} \mathbf{f}_{c(2)}^{R T} \mathbf{f}_{c(4)}^{C(3) T} \mathbf{f}_{c(4)}^{R T} \mathbf{f}_{c(4)}^{C(4) T}\right\}^{\mathrm{T}},
\end{aligned}
$$

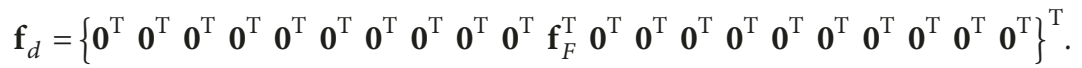




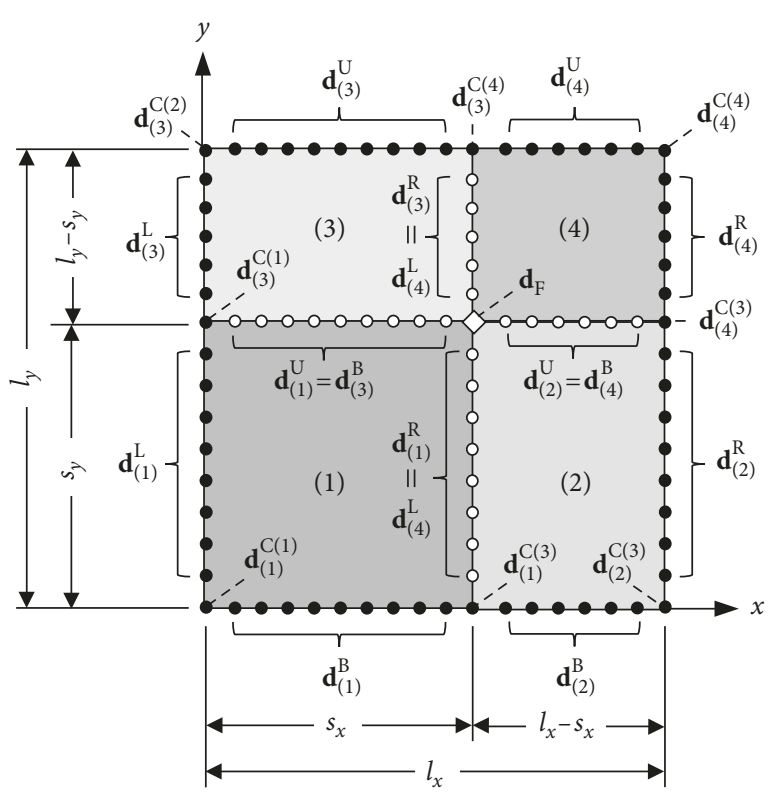

FIGURE 4: Four-element model for a rectangular plate subjected to a stationary point force $F_{k}$. $\diamond=$ the node at which the stationary point force $F_{k}$ is located, $\bullet$ = the nodes on the boundary edges, and $\mathrm{O}=$ the interim nodes without external loadings.

In (14), $\mathbf{S}_{(e)}(\omega)$ and $\mathbf{d}_{(e)}(e=1,2,3,4)$ are defined by (10) and (11), respectively; additionally, $\mathbf{T}_{(e)}$ are the $8\left(N_{x(e)}+\right.$ $\left.N_{y(e)}\right)$-by-12[( $\left.\left.N_{x(1)}+N_{x(2)}\right)+\left(N_{y(1)}+N_{y(3)}\right)-1\right]$ transformation matrices required for the assembly of four finite plate elements, and they satisfy the following relations:

$$
\mathbf{d}_{(e)}=\mathbf{T}_{(e)} \mathbf{d} \quad(e=1,2,3,4) .
$$

In (16) and (17), $\mathbf{0}$ denotes the zero vectors and $\mathbf{f}_{F}=$ $\left\{\begin{array}{llll}F_{k} & 0 & 0 & 0\end{array}\right\}^{\mathrm{T}}$.

3.3. Spectral Element Vibration Analysis. By imposing all geometric and natural boundary conditions on (13), and by using the same method commonly used in the standard FEM, we can obtain a reduced spectral element equation in the following form:

$$
\widetilde{S}(\omega) \widetilde{d}=\widetilde{f}_{c}+\widetilde{f}_{d},
$$

where the symbol $(\sim)$ denotes the reduced matrices and vectors after all geometric and natural boundary conditions are imposed.

To compute the natural frequencies, we consider the free vibration problem by removing all external force terms from (19). The natural frequencies are then determined by searching for the frequencies $\omega$ that satisfy the condition of $\operatorname{det}[\widetilde{S}(\omega)]=0$. As the matrix $\widetilde{S}(\omega)$ is a transcendental function of $\omega$, a proper root finding method [27] can be used to compute the natural frequencies.

By solving (19) for $\widetilde{\mathbf{d}}$, using the relation between $\widetilde{\mathbf{d}}$ and $\mathbf{d}$, and finally using the relations between $\mathbf{d}$ and $\mathbf{d}_{(e)}(e=1,2,3$, 4 ), the frequency-domain dynamic responses due to a stationary point force $F_{k}$ can be obtained as follows:

$$
W_{k}(x, y, \omega)= \begin{cases}\mathbf{N}_{(1)}(x, y, \omega) \mathbf{d}_{(1)}, & \left(0 \leq x \leq s_{x}, 0 \leq y \leq s_{y}\right), \\ \mathbf{N}_{(2)}(x, y, \omega) \mathbf{d}_{(2)}, & \left(s_{x} \leq x \leq l_{x}, 0 \leq y \leq s_{y}\right), \\ \mathbf{N}_{(3)}(x, y, \omega) \mathbf{d}_{(3)}, & \left(0 \leq x \leq s_{x}, s_{y} \leq y \leq l_{y}\right), \\ \mathbf{N}_{(4)}(x, y, \omega) \mathbf{d}_{(4)}, & \left(s_{x} \leq x \leq l_{x}, s_{y} \leq y \leq l_{y}\right),\end{cases}
$$

where

$$
\begin{array}{r}
\mathbf{N}_{(e)}(x, y, \omega)=\mathbf{N}\left(x, y, \omega ; N_{x(e)}, N_{y(e)}, l_{x(e)}, l_{y(e)}\right), \\
(e=1,2,3,4),
\end{array}
$$

where $\mathbf{N}(x, y, \omega)$ is the dynamic shape function matrix that is available from [29].

The above solution process is repeated to obtain the frequency-domain dynamic responses for all stationary point forces, $F_{k}(k=0,1,2, \ldots, K)$. Based on the superposition principle, the total frequency-domain dynamic responses due to all stationary point forces can be obtained from

$$
W(x, y, \omega)=\sum_{k=0}^{K} W_{k}(x, y, \omega) .
$$

Finally, the time-domain dynamic responses due to a moving point force can be obtained from (22), by using the inverse fast Fourier transform (IFFT) algorithm [35], as follows:

$$
w(x, y, t)=\operatorname{IFFT}[W(x, y, \omega)]
$$

\section{Vibration Analysis of a Multispan Plate Subjected to a Moving Point Force}

4.1. Frequency-Domain Representation of a Moving Point Force. Figure 5 shows a multispan rectangular plate (simply, multispan plate) with arbitrary supports and boundary conditions, where $(X, Y)$ denotes the global coordinates for the entire multispan plate. The total length and uniform width of the multispan plate are denoted by $L_{X}$ and $L_{Y}$, respectively, and the length of each span is denoted by $l_{x[q]}$, where $q=1,2,3, \ldots, Q$. The distance from the left edge of the first span $(X=0)$ to the right edge of the $q$-th span is denoted by $X_{[q]}$.

The multispan plate is subjected to a moving point force with a constant magnitude $P$, and the red dotted line in Figure 5 represents the arbitrary trajectory of the moving point force. The current position of the moving point force is denoted by $S_{X}(t)$ and $S_{Y}(t)$, with respect to the global coordinates $(X, Y)$, as follows:

$$
f(X, Y, t)=P \delta\left(X-S_{X}(t)\right) \delta\left(Y-S_{Y}(t)\right) .
$$

With the same approach used in Section 3.1, the moving point force represented by (24) can be transformed into the frequency domain as follows:

$$
\bar{f}(X, Y, \omega)=\sum_{k=0}^{K} F_{k} \delta\left(X-S_{X(k)}\right) \delta\left(Y-S_{Y(k)}\right),
$$




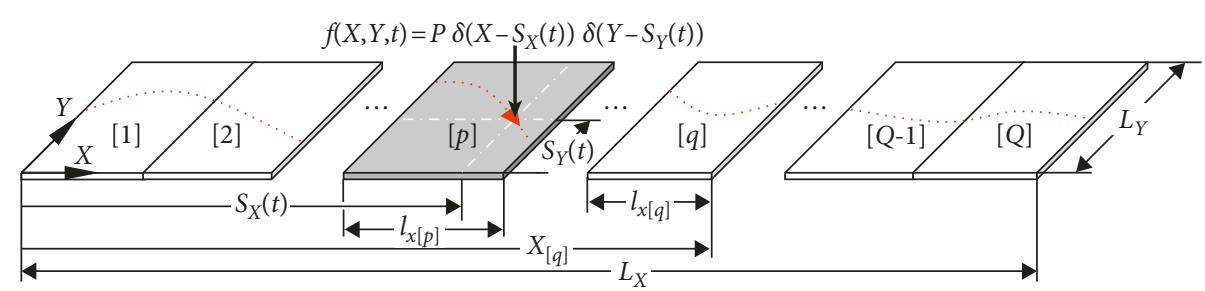

FIGURE 5: A multispan rectangular plate subjected to a moving point force $P$, where the red arrow indicates current position of the moving point force.

where $F_{k}(k=0,1,2, \ldots, K)$ is also defined by (8) and $K \leq N-1$. As illustrated in Figure 6, all stationary point forces are distributed along the trajectory of the moving point force. As shown in Figure 7, the position of the stationary point force $F_{k}$ with respect to the global coordinates $(X, Y)$ is denoted by $\left(S_{X(k)}, S_{Y(k)}\right)$, where

$$
X_{[p-1]} \leq S_{X(k)} \leq X_{[p]}, \quad 0 \leq S_{Y(k)} \leq L_{Y} .
$$

Similarly, the position of the stationary point force $F_{k}$ with respect to the local coordinates $(x, y)$ for the $p$-th span can be represented by $\left(s_{x(k)}, s_{y(k)}\right)$, where

$$
\begin{aligned}
& 0 \leq s_{x(k)} \leq l_{x[p]}, \\
& 0 \leq s_{y(k)} \leq L_{Y} .
\end{aligned}
$$

\subsection{Spectral Element Modeling for a Multispan Plate Subjected} to a Stationary Point Force. To obtain the frequency-domain vibration responses due to a stationary point force, $F_{k}$, which is located on the $p$-th span as shown in Figure 7, we should assemble one finite plate element on which the stationary point force $F_{k}$ is located (i.e., the $p$-th span) and $Q-1$ finite plate elements that are free from the stationary point force. By using the four-element model introduced in Section 3.2.2, the $p$-th span subjected to a stationary point force $F_{k}$ is represented by the four finite plate elements as shown in Figure $8(\mathrm{a})$. The stationary point force $F_{k}$ is placed at the junction $\left(s_{x}, s_{y}\right)$ of four finite plate elements. The subscripts $[p(e)](e=1,2,3,4)$ are used to denote each finite plate element number. For assembling with the $p$-th span represented by the four-element model, all other spans which are free from the stationary point force are represented by the two-element model as shown in Figure 8(b). The subscripts $[q(e)](e=1,2)$ are used to denote two finite plate elements for the $q$-th span, where $q \neq p$.
The spectral element equation for the $p$-th span subjected to a stationary point force can be readily obtained from (13) in the following form:

$$
\mathbf{S}_{[p]}(\omega) \mathbf{d}_{[p]}=\mathbf{f}_{c[p]}+\mathbf{f}_{d[p]} .
$$

The spectral element equations for all other spans which are free from the stationary point force can be derived by assembling the two finite plate elements shown in Figure 8(b) in the following form:

$$
\mathbf{S}_{[q]}(\omega) \mathbf{d}_{[q]}=\mathbf{f}_{c[q]}, \quad(q=1,2,3, \ldots, Q ; q \neq p) .
$$

Finally, by assembling all the spectral element equations given by (28) and (30), a global spectral element equation for the complete multi-span plate can be obtained as follows:

$$
\mathbf{S}^{g}(\omega) \mathbf{d}^{g}=\mathbf{f}_{c}^{\mathcal{g}}+\mathbf{f}_{d}^{\mathcal{g}} .
$$

4.3. Spectral Element Vibration Analysis. By imposing all geometric and natural boundary conditions on (30), we can obtain a reduced global spectral element equation in the following form:

$$
\widetilde{S}^{g}(\omega) \tilde{d}^{g}=\tilde{f}_{c}^{g}+\tilde{f}_{d}^{g}
$$

To compute natural frequencies, we consider free vibration problem by removing all external forcing terms from (31). As explained in Section 3.3, the natural frequencies are then computed from $\operatorname{det}\left[\widetilde{\mathbf{S}}^{g}(\omega)\right]=0$ by using a proper root finding method [27].

To obtain the frequency-domain dynamic responses, we first solve (31) for $\widetilde{\mathbf{d}}^{g}$ and then use the relations between $\widetilde{\mathbf{d}}^{g}$ and $\mathbf{d}^{g}$ and between $\mathbf{d}^{g}$ and $\boldsymbol{d}_{[q]}$ to obtain the nodal DOFs for the $q$-th span $(q=1,2,3, \ldots, Q)$. By using the relations between $\mathbf{d}_{[p]}$ and $\mathbf{d}_{[p(e)]}(e=1,2,3,4)$, the frequency-domain dynamic responses at the $p$-th span subjected to a stationary point force $F_{k}$ can be obtained, in the local coordinates $(x, y)$, as follows:

$$
W_{k[p]}(x, y, \omega)= \begin{cases}\mathbf{N}_{[p(1)]}(x, y, \omega) \mathbf{d}_{[p(1)]}, & \left(0 \leq x \leq s_{x}, 0 \leq y \leq s_{y}\right), \\ \mathbf{N}_{[p(2)]}(x, y, \omega) \mathbf{d}_{[p(2)]}, & \left(s_{x} \leq x \leq l_{x[p]}, 0 \leq y \leq s_{y}\right), \\ \mathbf{N}_{[p(3)]}(x, y, \omega) \mathbf{d}_{[p(3)]}, & \left(0 \leq x \leq s_{x}, s_{y} \leq y \leq L_{Y}\right), \\ \mathbf{N}_{[p(4)]}(x, y, \omega) \mathbf{d}_{[p(4)]}, & \left(s_{x} \leq x \leq l_{x[p]}, s_{y} \leq y \leq L_{Y}\right),\end{cases}
$$




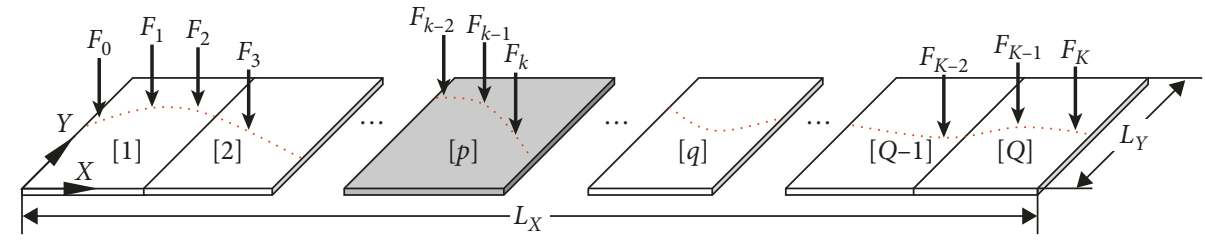

FIGURE 6: Frequency-domain representation of a moving point force on the multispan plate.

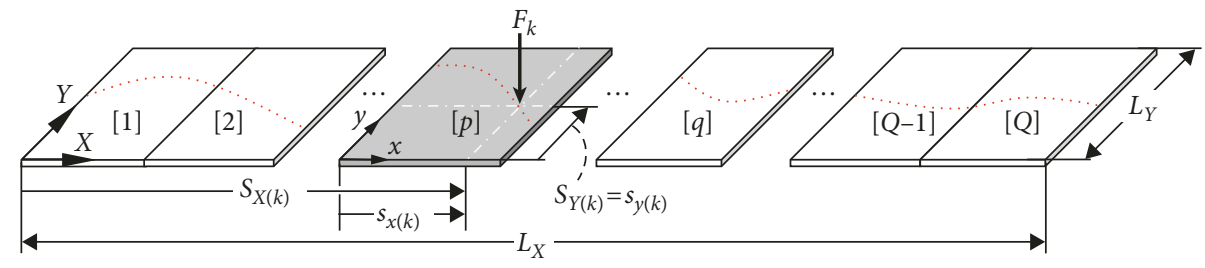

FIgURE 7: A multispan rectangular plate subjected to a stationary point force $F_{k}$ acting on the $p$-th span.

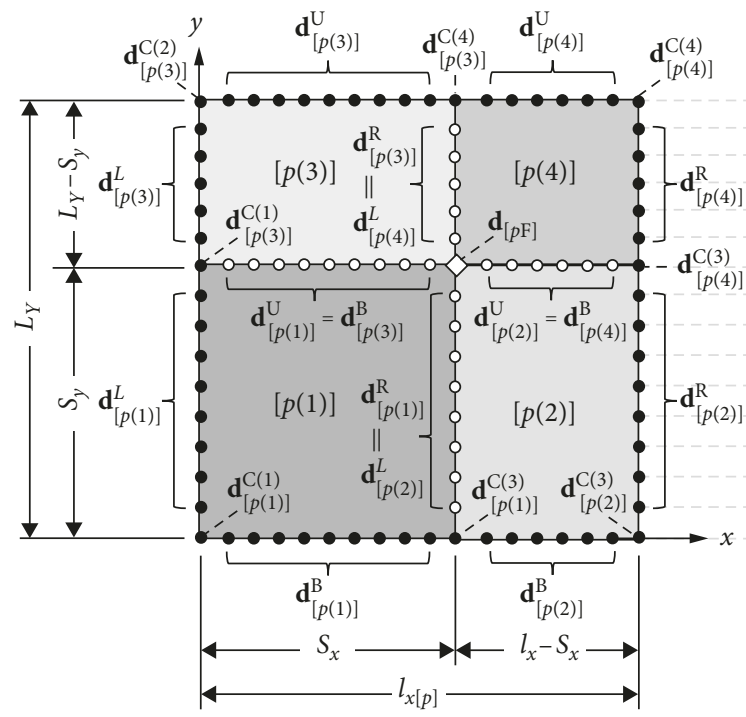

(a)

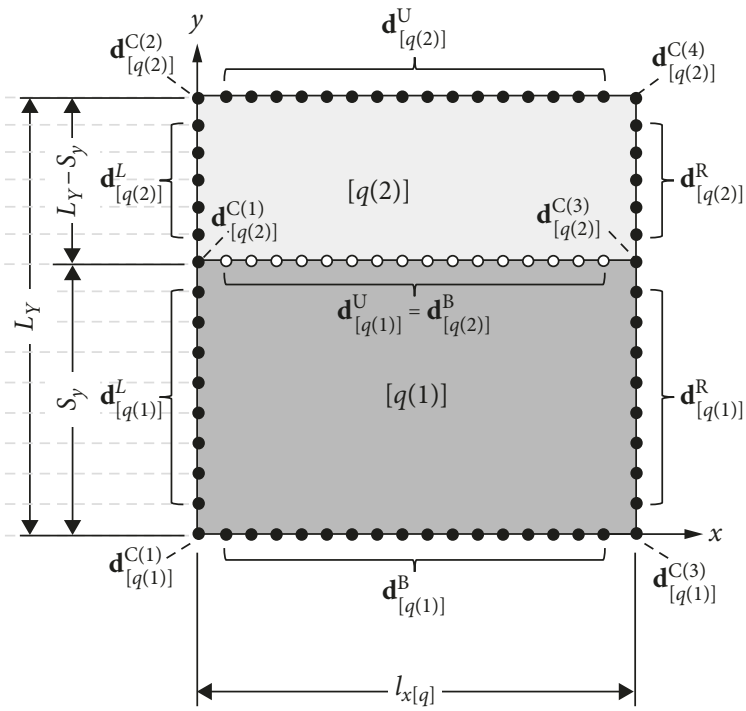

(b)

Figure 8: (a) Four-element model for the span subjected to a stationary point force $F_{k}$ and (b) two-element model for the spans that are free from the stationary point force. $\diamond=$ the node at which the stationary point force $F_{k}$ is located, $\bullet=$ the nodes on the boundary edges, and $\mathrm{O}=$ the interim nodes without external loadings. (a) When $q=p$, (b) when $q \neq p$.

where $\boldsymbol{N}_{[p(e)]}(x, y, \omega)$ denotes the dynamic shape functions defined by (21) for the element $[p(e)]$. Similarly, by using the relation between $\mathbf{d}_{[q]}$ and $\mathbf{d}_{[q(e)]}(e=1,2)$, the frequency-domain dynamic responses in the q-th span, which is free from the stationary point force, can be also obtained as follows:

$$
W_{k[q]}(x, y, \omega)= \begin{cases}\mathbf{N}_{[q(1)]}(x, y, \omega) \mathbf{d}_{[q(1)]}, & \left(0 \leq x \leq l_{x[q]}, 0 \leq y \leq s_{y}\right), \\ \mathbf{N}_{[q(2)]}(x, y, \omega) \mathbf{d}_{[q(2)]}, & \left(0 \leq x \leq l_{x[q]}, s_{y} \leq y \leq L_{Y}\right),\end{cases}
$$

By using (32) and (33), the frequency-domain vibration responses of the entire multispan plate due to a stationary point force $F_{k}$ can be represented with respect to the global coordinates $(X, Y)$ as follows:

$$
W_{k}(x, y, \omega)=\sum_{q=1}^{Q} W_{k[q]}(x, y, \omega) H_{[q]}(X),
$$


where

$$
H_{[q]}(X)=h\left(X-X_{[q-1]}\right)-h\left(X-X_{[q]}\right),
$$

where $h(X)$ denotes the Heaviside step function defined by [34]

$$
h(X)= \begin{cases}0, & (X<0) \\ 1, & (X \geq 0),\end{cases}
$$

The above solution process is repeated to obtain the frequency-domain vibration responses for all stationary point forces. By using the superposition principle, the total frequency-domain dynamic responses can be obtained as follows:

$$
W(x, y, \omega)=\sum_{k=0}^{K} W_{k}(x, y, \omega) .
$$

Finally, the time-domain dynamic responses can be obtained from (37), by using the IFFT algorithm, as follows:

$$
w(X, Y, t)=\operatorname{IFFT}[W(x, y, \omega)]
$$

\section{Numerical Results and Discussions}

For the numerical study, three single-span square plates and two three-span plates subjected to a moving point force $P=1 \mathrm{~N}$ are considered as example problems. All example plates have null initial conditions. As shown in Figure 9, the three examples of square plates have different boundary conditions as follows: (1) Example 1: simplesimple-simple-simple (S-S-S-S) boundary condition; (2) Example 2: simple-free-simple-free (S-F-S-F) boundary condition; and (3) Example 3: clamped-free-free-free (C-F-F-F) boundary condition. As shown in Figure 10, the two examples of three-span rectangular plates have free boundary conditions at the two parallel edges at $Y=0$ and $L$, but they have different supports at $X=0, L, 3 L$, and $4 L$ as follows: (1) Example 4: simple-simple-simple-simple (S-S-S-S) supports and (2) Example 5: clamped-simple-simple-simple (C-S-S-S) supports.

The three examples of square plates (Examples 1, 2, and 3 ) and two examples of three-span plates (Examples 4 and 5) have a uniform thickness $h=0.001 \mathrm{~m}$. Examples 1, 2 , and 3 have the same dimensions of $L=1 \mathrm{~m}$. Examples 4 and 5 have the same width $L=1 \mathrm{~m}$ and the same lengths of spans given by $L, 2 L$, and $L$, as shown in Figure 10 .

The material properties of Examples 1, 2 and 3 are given as follows: Young's modulus $E=69 \times 10^{9} \mathrm{~N} / \mathrm{m}^{2}$, Poisson's ratio $\nu=0.33$, and mass density $\rho=2700 \mathrm{~kg} / \mathrm{m}^{3}$. The material properties for the first and third spans of Examples 4 and 5 are identical to those for Examples 1,2 , and 3. However, the material properties for the second span of Examples 4 and 5 are assumed to have different values as follows: $E=207 \times 10^{9} \mathrm{~N} / \mathrm{m}^{2}, \nu=0.33$, and $\rho=2700 \mathrm{~kg} / \mathrm{m}^{3}$.

For Examples 1, 2, and 3, three cases of different trajectories of a moving point force are considered, which are represented with respect to the coordinates $(x, y)$ as follows:

$$
\begin{array}{ll}
s_{x}(t)=v_{0} t, s_{y}(t)=\frac{1}{2} L & \text { (Case 1), } \\
s_{x}(t)=v_{0} t, s_{y}(t)=v_{0} t & \text { (Case 2), } \\
s_{x}(t)=v_{0} t, s_{y}(t)=\frac{1}{2} L\left[1+\frac{1}{2} \sin \left(2 \pi v_{0} t / L\right)\right] & (\text { Case 3). }
\end{array}
$$

Here, $v_{0}$ denotes a constant moving speed. Case 1 represents the case where the moving point force travels along the straight centerline of the plate, Case 2 represents the case where the moving point force crosses the catercornered plate, and Case 3 represents the case where the moving point force travels along a sinusoidal trajectory with respect to the centerline of the plate. For Examples 4 and 5, the moving point force is assumed to travel along the straight centerline of the multispan plates, which can be represented with respect to the global coordinates $(X, Y)$ as follows:

$$
\begin{aligned}
& S_{X}(t)=v_{0} t, \\
& S_{Y}(t)=\frac{1}{2} L .
\end{aligned}
$$

5.1. Evaluation of the Proposed Method. It is necessary to verify the proposed method. Thus, the accuracy and computational efficiency of the proposed SEM were evaluated by comparing the natural frequencies and vibration responses with those obtained by other solution techniques: the exact theory [37], ITM [5], FEM [14, 38], and the well-known commercial finite element analysis package ANSYS [39].

For the evaluation of the proposed SEM, four example problems are chosen from Figures 9 and 10, as follows: (1) Example 1, where the trajectory of the moving point force is given by Case 1 of (39); (2) Example 1, where the trajectory of moving point force is given by Case 2 of (39); (3) Example 1 , where the trajectory of moving point force is given by Case 3 of (39); and (4) Example 4, where the trajectory of moving point force is given by (40).

For the results obtained using the FEM, a four-node 12-DOF (nonconforming) finite rectangular thin plate element model (known as ACM element) [38] was used. The vibration responses by the FEM were obtained using the technique introduced by $\mathrm{Wu}$ et al. [14] for moving force problems.

Table 1 compares the lowest first ten natural frequencies of the S-S-S-S square plate (Example 1) obtained by the exact theory [37], FEM, and SEM. The exact natural frequencies (in $\mathrm{Hz}$ ) of a thin S-S-S-S classical (or Kirchhoff) plate are given by [37]

$$
f_{(m, n)}=\frac{\pi\left(m^{2}+n^{2}\right)}{2 L^{2}} \sqrt{\frac{D}{\rho h}},
$$

where the subscripts $(m, n)$ denote the mode number. In the FEM, the natural frequencies are determined by using MATLAB function "eigs" [40]. For the proposed SEM, a single finite element is used for the entire single-span plate, and the root-finding method in [27] is applied to obtain the 


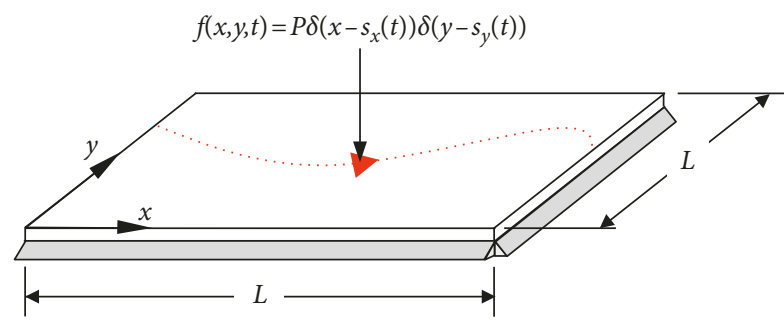

(a)

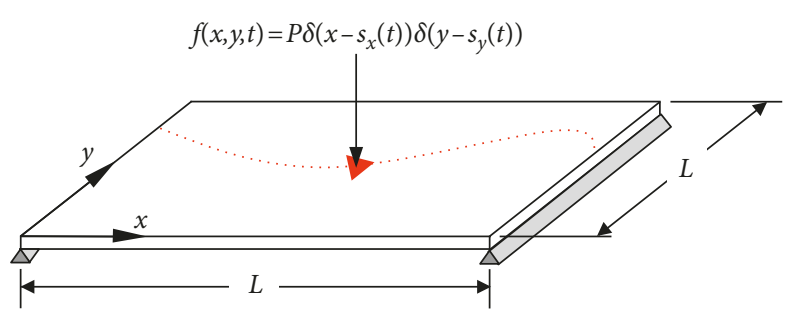

(b)

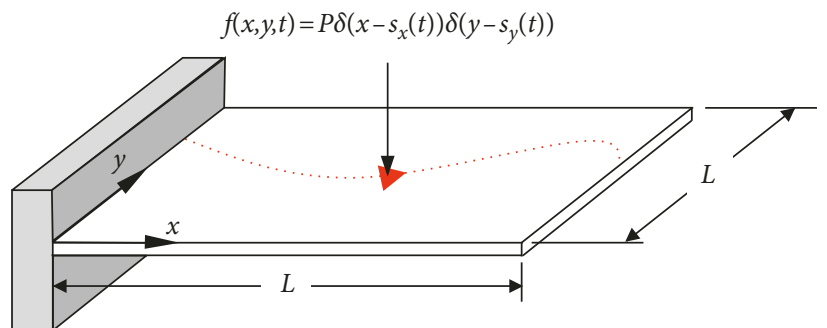

(c)

Figure 9: Three examples of square plates subjected to a moving point force $P$, where the red arrow indicates current position of the moving point force. (a) Example 1: S-S-S-S boundary condition. (b) Example 2: S-F-S-F boundary condition. (c) Example 3: C-F-F-F boundary condition.

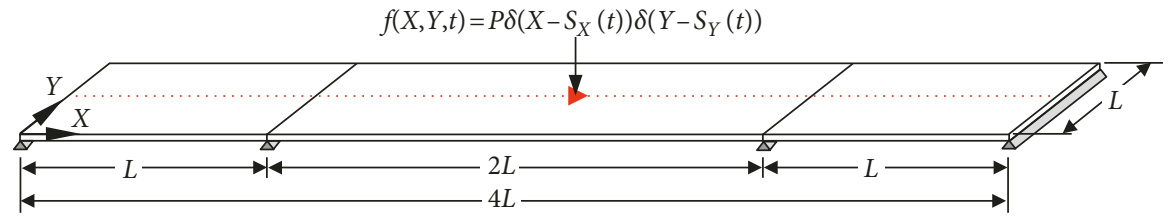

(a)

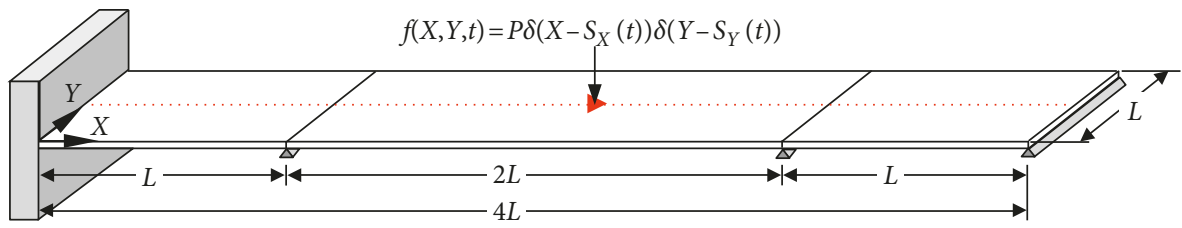

(b)

Figure 10: Two examples of three-span rectangular plates subjected to a moving point force $P$, where the red arrow indicates current position of the moving point force. (a) Example 4: S-S-S-S supports. (b) Example 5: C-S-S-S supports.

TABLE 1: Comparison of the natural frequencies (Hz) of the S-S-S-S single-span square plate (Example 1) obtained by the exact theory [37], FEM [38], and SEM.

\begin{tabular}{lccccc}
\hline Mode number $(m, n)$ & Exact & & $\begin{array}{c}\text { FEM }\left(n_{\mathrm{E}}, n_{\mathrm{D}}\right) \\
n_{\mathrm{E}}=10 \times 10, n_{\mathrm{D}}=279\end{array}$ & $\begin{array}{c}\text { SEM }\left(n_{\mathrm{E}}, n_{\mathrm{D}}\right) \\
n_{\mathrm{E}}=50 \times 50, n_{\mathrm{D}}=7399\end{array}$ & $\begin{array}{c}n_{\mathrm{E}}=100 \times 100, n_{\mathrm{D}}=29799 \\
n_{\mathrm{E}}=1 \times 1, n_{\mathrm{D}}=636\end{array}$ \\
\hline$(1,1)$ & 4.857 & 4.831 & 4.856 & 4.856 & 4.857 \\
$(1,2)$ & 12.14 & 12.04 & 12.14 & 12.14 & 12.14 \\
$(2,2)$ & 19.43 & 19.03 & 19.41 & 24.42 & 19.43 \\
$(1,3)$ & 24.28 & 24.07 & 24.27 & 31.56 & 24.28 \\
$(2,3)$ & 31.57 & 30.72 & 31.53 & 41.28 & 41.57 \\
$(1,4)$ & 41.28 & 40.97 & 41.26 & 43.69 & 41.28 \\
$(3,3)$ & 43.71 & 41.87 & 43.62 & 48.55 & 43.71 \\
$(2,4)$ & 48.57 & 47.17 & 48.50 & 60.67 & 60.71 \\
$(3,4)$ & 60.71 & 57.63 & 60.56 & 63.13 & 63.14 \\
$(1,5)$ & 63.14 & 62.82 & 63.11 & & \\
\hline
\end{tabular}

Note. $n_{\mathrm{E}}=$ total number of finite elements and $n_{\mathrm{D}}=$ total number of DOFs. 
natural frequencies. Table 1 shows that the SEM results are almost identical to the exact solutions given by (41). Table 1 also shows that the FEM results converge to both the exact solutions and the SEM results as the number of finite elements used in FEM is increased to more than $100 \times 100$.

Table 2 compares the lowest first ten natural frequencies of the three-span rectangular plate with S-S-S-S supports (Example 4) obtained by the FEM, ANSYS, and SEM. The finite element model created in ANSYS is shown in Figure 11. The ANSYS finite element model is obtained by using the SHELL63 element that is a four-node finite rectangular plate element model based on the classical (Kirchhoff) plate theory [39]. For the ANSYS results, the MODAL [39] is chosen as the type of analysis. The MODAL in ANSYS performs a modal analysis. For the SEM results, only three finite elements are used for the entire three-span plate (i.e., a single finite element for each span). Table 2 shows that the FEM results converge to the SEM results as more finite elements are employed in the FEM analysis. Table 2 also shows that the ANSYS results obtained by using a sufficient number of finite elements (e.g., $400 \times 100$ finite elements) are found to be identical to the SEM results.

Figure 12 compares the vibration responses for $0 \leq t \leq T_{\mathrm{A}}=0.2 \mathrm{~s}$ at the center $(x=y=0.5 \mathrm{~L})$ of the S-S-S-S square plate (Example 1) obtained using the ITM [5], FEM [14], and SEM, when the trajectory of the moving point force is given by Case 1 of (39) with $v_{0}=5 \mathrm{~m} / \mathrm{s}$. The vibration responses obtained by the ITM [5] are given by

$$
\begin{aligned}
w(x, y, t)= & \frac{4 P}{\rho h L^{2}} \sum_{m=1}^{\infty} \sum_{n=1}^{\infty} \frac{\sin (n \pi / 2) \sin (m \pi x / L) \sin (n \pi y / L)}{\left(2 \pi f_{(m, n)}\right)^{2}-n^{2} \pi^{2} v_{0}^{2} / L^{2}} \\
& \cdot \sin \frac{m \pi v_{0} t}{L}-\frac{4 P}{\rho h L^{2}} \sum_{m=1}^{\infty} \sum_{n=1}^{\infty} \\
& \cdot \frac{\sin (n \pi / 2) \sin (m \pi x / L) \sin (n \pi y / L)}{2 \pi f_{(m, n)}\left[\left(2 \pi f_{(m, n)}\right)^{2}-n^{2} \pi^{2} v_{0}^{2} / L^{2}\right]} \frac{m \pi v_{0}}{L} \\
& \cdot \sin 2 \pi f_{(m, n)} t .
\end{aligned}
$$

Figure 12(a) shows that the vibration responses obtained by the ITM converge gradually to the SEM results when the total number of vibration modes used in the ITM-based analysis exceeds 100. Figure 12(b) also shows that the vibration responses obtained by the FEM converge gradually to the SEM results as the total number of finite elements used in the FEM-based analysis is increased to more than $40 \times 40$.

Figure 13 compares the vibration responses for $0 \leq t \leq T_{\mathrm{A}}=$ $0.2 \mathrm{~s}$ at the center $(x=y=0.5 \mathrm{~L})$ of the S-S-S-S square plate (Example 1) obtained by the present SEM and FEM for two different trajectories of a moving point force $P=1 \mathrm{~N}$ : (a) Case 2 of (39), where $v_{0}=5 \mathrm{~m} / \mathrm{s}$, and (b) Case 3 of (39), where $v_{0}=5 \mathrm{~m} / \mathrm{s}$. Figure 13 clearly shows that, for both Case 2 and Case 3, the vibration responses obtained by the FEM converge gradually to the SEM results as the total number of finite elements used in the FEM-based analysis is increased to more than $40 \times 40$.
Figure 14 compares the vibration responses for $0 \leq t \leq T_{\mathrm{A}}=$ $0.2 \mathrm{~s}$ at the center $(x=y=0.5 \mathrm{~L})$ of the $\mathrm{S}-\mathrm{S}-\mathrm{S}-\mathrm{S}$ square plate (Example 1) obtained by the present SEM and ANSYS [39] for the same moving point force $P=1 \mathrm{~N}$ as considered in Figure 13. The moving point force travels along two different trajectories: (a) Case 2 of (39), where $v_{0}=5 \mathrm{~m} / \mathrm{s}$, and (b) Case 3 of (39), where $v_{0}=5 \mathrm{~m} / \mathrm{s}$. For the ANSYS results, the finite element model for the S-S-S-S square plate (Example 1) is obtained by using the SHELL63 element in ANSYS [39]. The technique proposed by $\mathrm{Wu}$ et al. [14] is then used to represent a moving point force in the ANSYS finite element model. For the ANSYS simulations, the TRANS [39] is chosen as the type of analysis. The TRANS in ANSYS performs a transient dynamic analysis. Similar to Figure 13, Figure 14 also shows that the ANSYS results converge gradually to the SEM results as the total number of finite elements used in the ANSYS analysis is increased to more than $40 \times 40$.

Figure 15 compares the vibration responses obtained by the FEM and SEM for $0 \leq t \leq T_{A}=0.8 \mathrm{~s}$ at three locations, that is, at the center of the first span $(X=0.5 L, Y=0.5 L)$, at the center of the second $\operatorname{span}(X=2 L, Y=0.5 L)$, and at the center of the last $\operatorname{span}(X=3.5 L, Y=0.5 L)$ of the $S-S-S-S$ three-span rectangular plate (Example 4 ) subjected to a moving point force whose trajectory is given by (40) where $v_{0}=5 \mathrm{~m} / \mathrm{s}$. Figure 15 also clearly shows that the FEM results approach the SEM results as the number of finite elements used in the FEM-based analysis is increased to more than $160 \times 40$.

The CPU times required to compute the SEM and FEM results shown in Figure 15 are measured with a standard workstation PC equipped with two sockets of Intel Xeon E5$2630 \mathrm{v} 3$ processors clocked at $2.4 \mathrm{GHz}$, and $384 \mathrm{~GB}$ of DDR4 RAM memory. For the SEM analysis, the span subjected to a stationary point force defined by (8) is represented by the four finite elements as shown in Figure 8(a), and each finite element is represented by 20 finite strip elements in both $x$ and $y$-directions. However, the span that is free from the stationary point force is divided into two finite elements as shown in Figure 8(b) and each finite element is represented by 40 finite strip elements in the $x$-direction and 20 finite strip elements in the $y$-direction. The total number of stationary point forces considered for the S-S-S-S three-span rectangular plate (Example 4) is 64. For the FEM analysis, the example S-S-S-S three-span rectangular plate is represented by $40 \times 10,80 \times 20$, and $160 \times 40$ finite elements, and the FEM results are obtained using the MATLAB function “ode45" [40]. The CPU time for the SEM results obtained by using $4 \times 2$ finite elements is approximately $1.5 \mathrm{~h}$, while the CPU times required for the FEM results obtained by using $40 \times 10$ finite elements, $80 \times 20$ finite elements, and $160 \times 40$ finite elements are $0.1 \mathrm{~h}, 5.5 \mathrm{~h}$, and $305 \mathrm{~h}$, respectively.

Based on the above observations, we can conclude that the proposed SEM provides highly accurate solutions and demands a relatively smaller computational cost when compared with the standard FEM.

5.2. Effects of the Boundary Conditions and Moving Speed on the Vibration Responses. It has been well known that the vibration responses of a structure are dependent on its 
TABLE 2: Comparison of the natural frequencies (Hz) of the three-span plate with S-S-S-S supports (Example 4) obtained by the FEM [38], ANSYS [39], and SEM.

\begin{tabular}{|c|c|c|c|c|c|}
\hline $\begin{array}{l}\text { Mode } \\
\text { number }\end{array}$ & $n_{\mathrm{E}}=40 \times 10, n_{\mathrm{D}}=1265$ & $\begin{array}{c}\text { FEM }\left(n_{\mathrm{E}}, n_{\mathrm{D}}\right) \\
n_{\mathrm{E}}=120 \times 30, n_{\mathrm{D}}=11005\end{array}$ & $n_{\mathrm{E}}=480 \times 120, n_{\mathrm{D}}=173635$ & $\begin{array}{c}\text { ANSYS }\left(n_{\mathrm{E}}, n_{\mathrm{n}}\right) \\
n_{\mathrm{E}}=400 \times 100, n_{\mathrm{n}}=40501\end{array}$ & $\begin{array}{c}\operatorname{SEM}\left(n_{\mathrm{E}}, n_{\mathrm{D}}\right) \\
n_{\mathrm{E}}=3 \times 1, n_{\mathrm{D}}=1584\end{array}$ \\
\hline 1 & 1.268 & 1.267 & 1.267 & 1.267 & 1.267 \\
\hline 2 & 3.013 & 3.008 & 3.007 & 3.007 & 3.007 \\
\hline 3 & 3.019 & 3.018 & 3.018 & 3.018 & 3.018 \\
\hline 4 & 3.471 & 3.465 & 3.465 & 3.465 & 3.465 \\
\hline 5 & 4.595 & 4.594 & 4.594 & 4.594 & 4.594 \\
\hline 6 & 4.814 & 4.813 & 4.813 & 4.813 & 4.813 \\
\hline 7 & 5.026 & 5.017 & 5.016 & 5.016 & 5.016 \\
\hline 8 & 7.347 & 7.345 & 7.345 & 7.345 & 7.345 \\
\hline 9 & 9.388 & 9.362 & 9.359 & 9.359 & 9.359 \\
\hline 10 & 9.433 & 9.481 & 9.487 & 9.487 & 9.487 \\
\hline
\end{tabular}

Note. $n_{\mathrm{E}}=$ total number of finite elements, $n_{\mathrm{D}}=$ total number of DOFs, and $n_{\mathrm{n}}=$ total number of nodes.

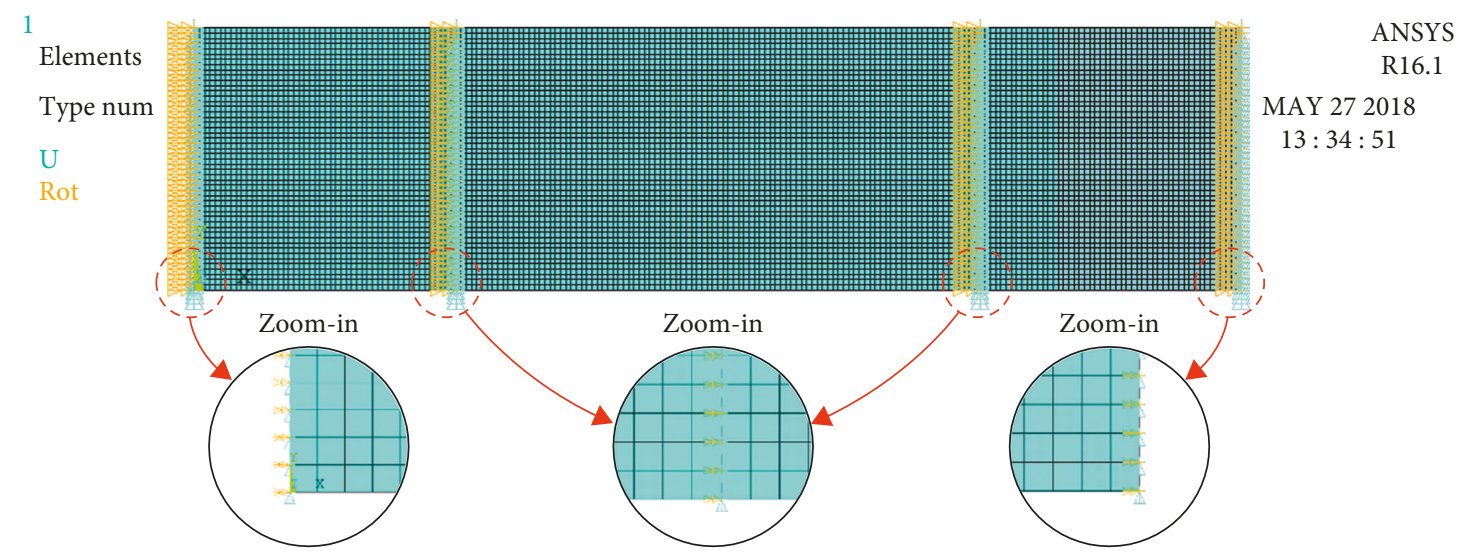

FIgURE 11: The finite element model created in ANSYS for evaluating natural frequencies of the three-span rectangular plate with S-S-S-S supports (Example 4).

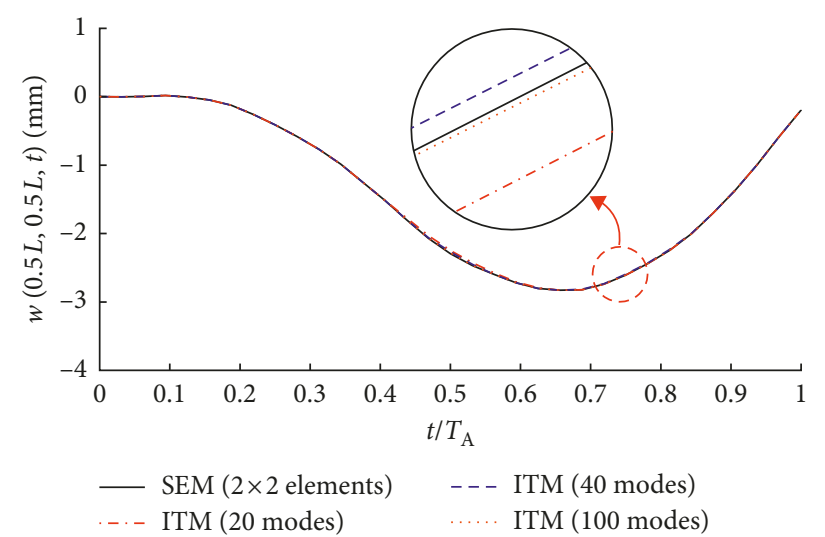

(a)

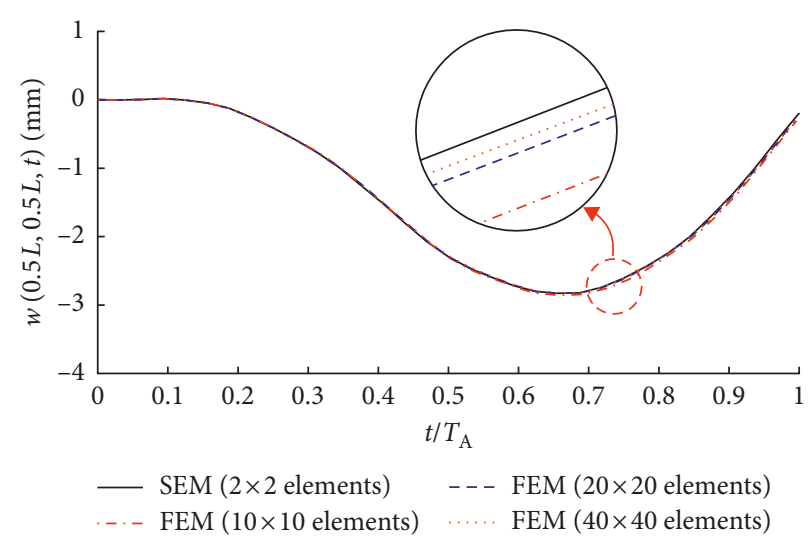

(b)

FIGURE 12: Comparison of the vibration responses obtained by the integral transform method (ITM) [5], FEM [14], and SEM at the center of the S-S-S-S square plate (Example 1) subjected to a moving point force whose trajectory is given by Case 1 of $(39)$, where $v_{0}=5 \mathrm{~m} / \mathrm{s}$. (a) ITM [5] versus SEM. (b) FEM [14] versus SEM.

boundary conditions and external loading. Since the high accuracy of the proposed SEM was verified in the previous section, we applied it to square plates with three different boundary conditions (Examples 1-3 in Figure 9) and threespan rectangular plates with two different boundary conditions (Examples 4-5 in Figure 10) to investigate the effects of boundary conditions on the vibration responses of example plates depending on the speed of a moving point force. Three types of moving force trajectories given by (39) are considered for the example square plates, while a single 


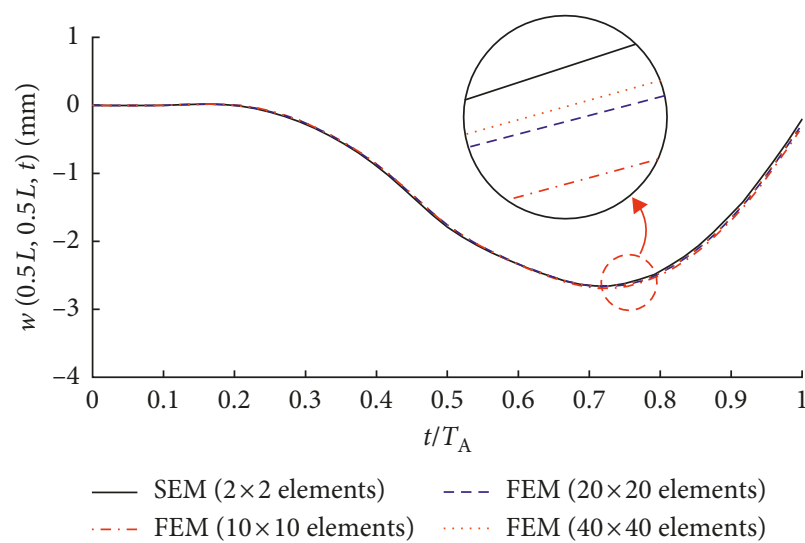

(a)

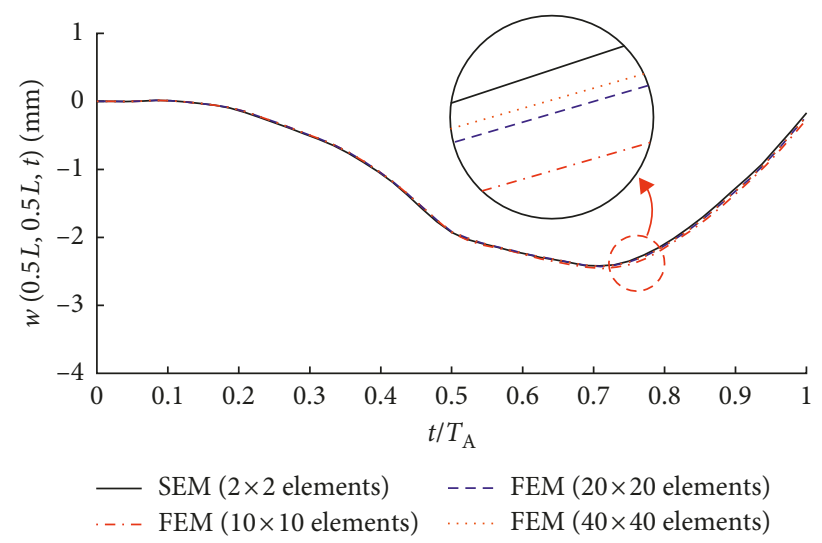

(b)

FIGURE 13: Comparison of the vibration responses obtained by the FEM [14] and SEM at the center of the S-S-S-S square plate (Example 1) subjected to a moving point force whose trajectory is given by (a) Case 2 of (39), where $v_{0}=5 \mathrm{~m} / \mathrm{s}$ and (b) Case 3 of ( 39 ), where $v_{0}=5 \mathrm{~m} / \mathrm{s}$.

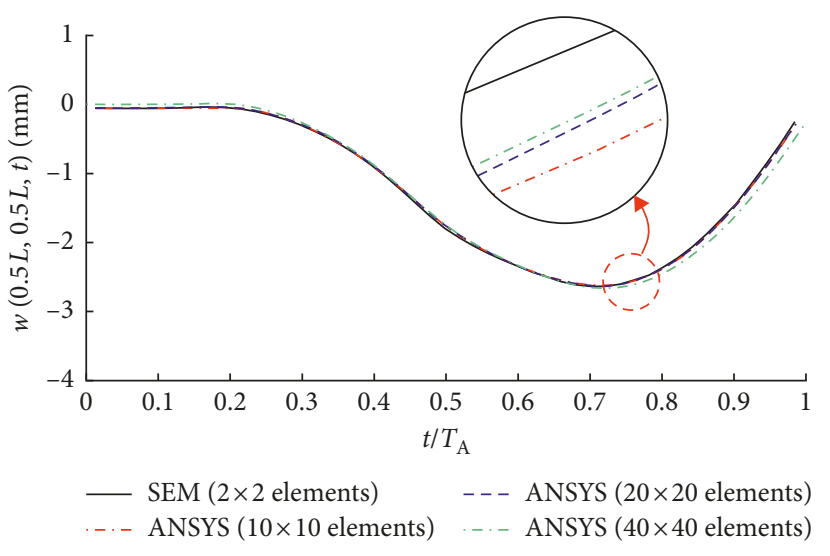

(a)

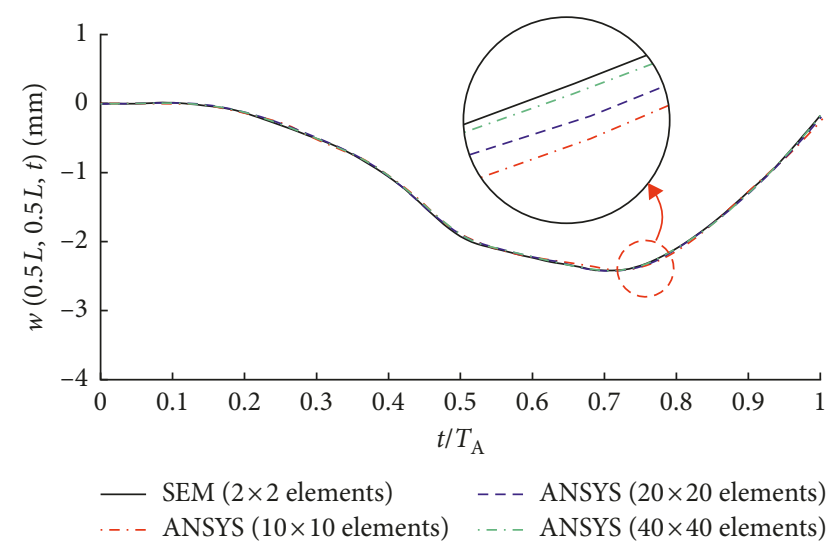

(b)

FIgURE 14: Comparison of the vibration responses obtained by the ANSYS [39] and SEM at the center of the S-S-S-S square plate (Example 1) subjected to a moving point force whose trajectory is given by (a) Case 2 of (39), where $v_{0}=5 \mathrm{~m} / \mathrm{s}$, and (b) Case 3 of (39), where $v_{0}=5 \mathrm{~m} / \mathrm{s}$.

moving force trajectory given by (40) is considered for the example three-span rectangular plates.

Figures 16-18 show the effects of boundary conditions and moving speed $v_{0}$ on the vibration responses at the center $(x=y=0.5 L)$ of the S-S-S-S (Example 1), S-F-S-F (Example 2), and C-F-F-F (Example 3) square plates when a moving point force travels along the trajectories given by Case 1, Case 2, and Case 3 of (39).

From Figures 16-18, the vibration amplitudes are found to be quite dependent on the boundary conditions and the moving speed $v_{0}$ as follows: (1) The vibration amplitude of the C-F-F-F plate (Example 3 ) tends to decrease as the moving speed $v_{0}$ decreases, and (2) the S-S-S-S plate (Example 1) seems to have the smaller vibration amplitude than the S-F-S-F (Example 2) for all assumed trajectories of a moving point force.

When a moving point force travels on the S-S-S-S plate (Example 1) at a constant speed $v_{0}$ along the trajectories given by Case 1 of (39), the lowest critical speed can be obtained by equating the period of the $(1,1)$ mode to the time needed to pass through a length double that of the plate length, as follows [5]:

$$
v_{c r}=2 f_{11} L=9.713 \mathrm{~m} / \mathrm{s}
$$

Figure 19 shows the effects of moving speed on the vibration responses at three locations of the example S-S-S-S square plate: $(x=0.25 L, y=0.5 L), \quad(x=y=0.5 L)$, and $(x=0.75 L, y=0.5 L)$. From Figure 19 , we can find the following: (1) When $v_{0}<v_{\mathrm{cr}}$, the vibration amplitudes tend to return to the original undeformed state; (2) when $v_{0} \geq v_{\mathrm{cr}}$, the vibration amplitudes tend to keep increasing to have a maximum value until the moving force reaches the other edge of the plate; (3) the maximum deflection of the plate when $v_{0}<v_{\mathrm{cr}}$ is slightly larger than that when $v_{0} \geq v_{\mathrm{cr}}$; and (4) the maximum deflection of the plate tends to decrease when $v_{0}$ becomes larger than $v_{\mathrm{cr}}$. These findings appear quite similar to those obtained for the simply supported beam subjected to a moving point force in [5]. 


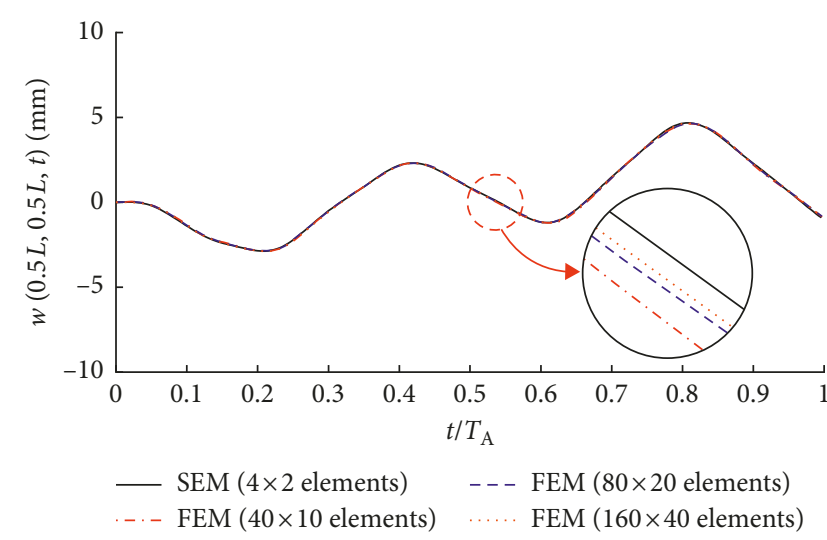

(a)

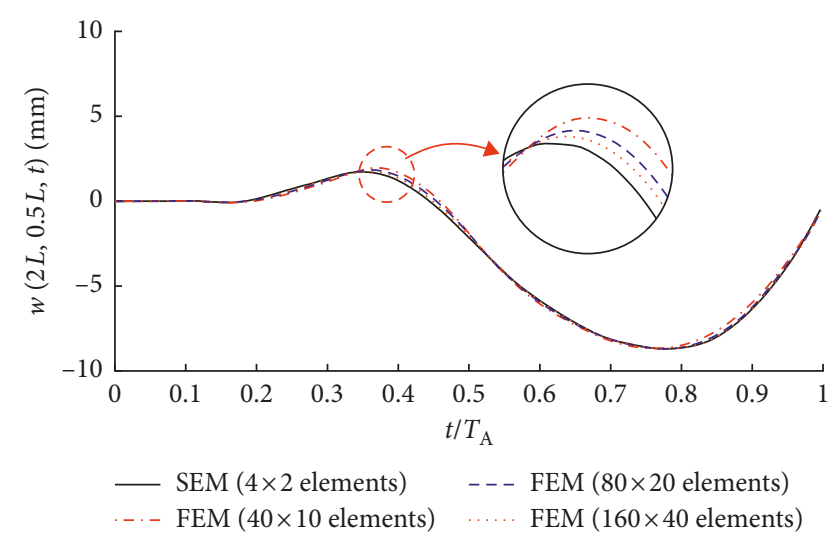

(b)

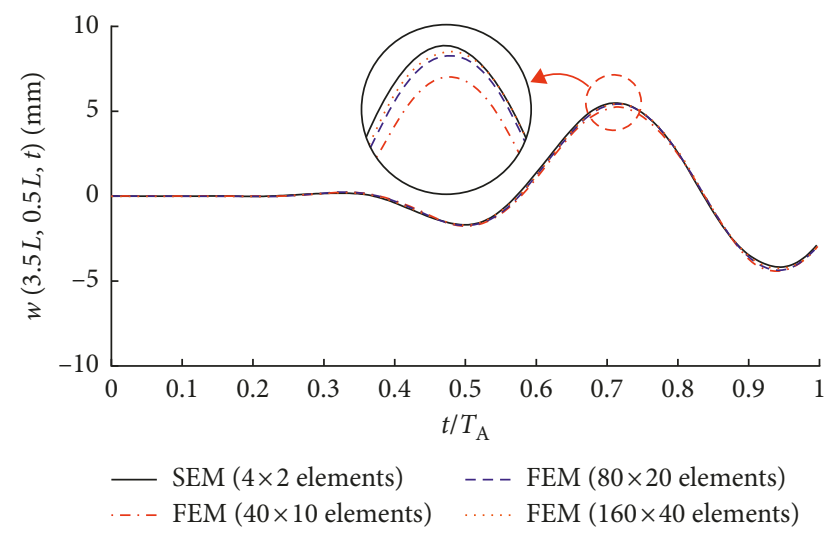

(c)

Figure 15: Comparison of the vibration responses obtained by the FEM [14] and SEM at three different locations of the S-S-S-S three-span rectangular plate (Example 4) subjected to a moving point force whose trajectory is given by (40), where $v_{0}=5 \mathrm{~m} / \mathrm{s}$. (a) At $X=0.5 \mathrm{~L}, Y=0.5 \mathrm{~L}$, (b) at $X=2 L, Y=0.5 L$, and (c) at $X=3.5 L, Y=0.5 L$.

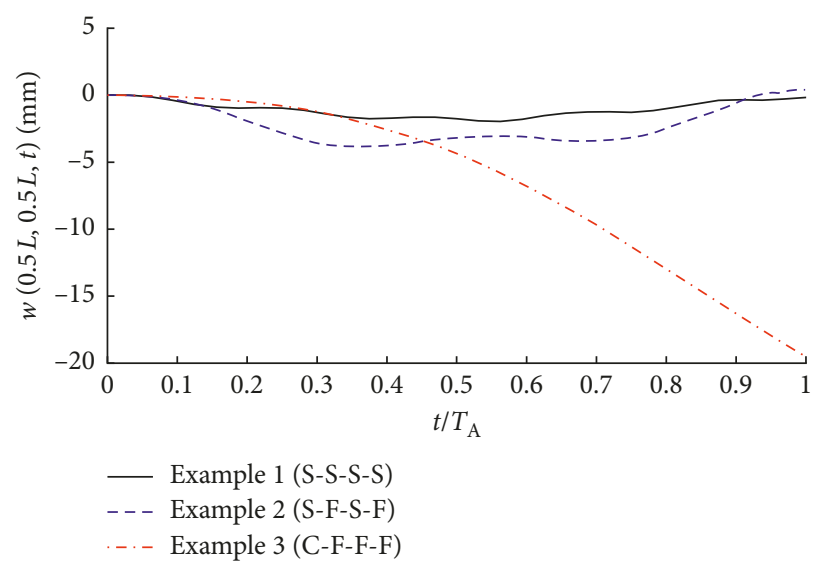

(a)

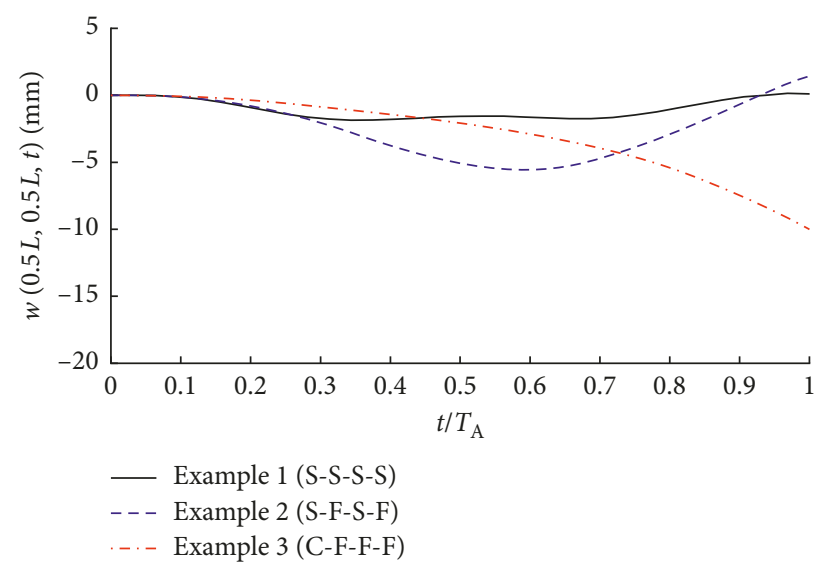

(b)

Figure 16: Continued. 


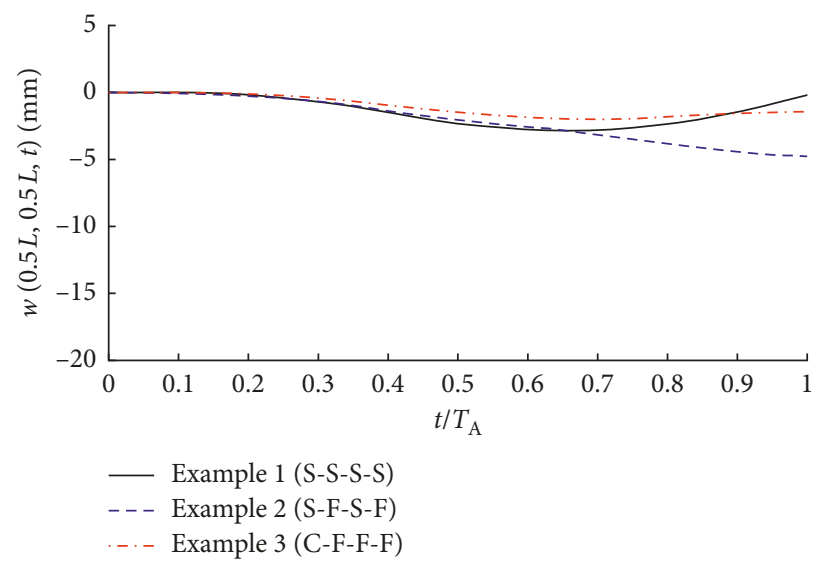

(c)

Figure 16: Effects of boundary conditions and moving speed $v_{0}$ on the vibration responses at the center of three square plates when a moving point force travels along the trajectory given by Case 1 of (39). (a) $v_{0}=1 \mathrm{~m} / \mathrm{s}$, (b) $v_{0}=2 \mathrm{~m} / \mathrm{s}$, and (c) $v_{0}=5 \mathrm{~m} / \mathrm{s}$.

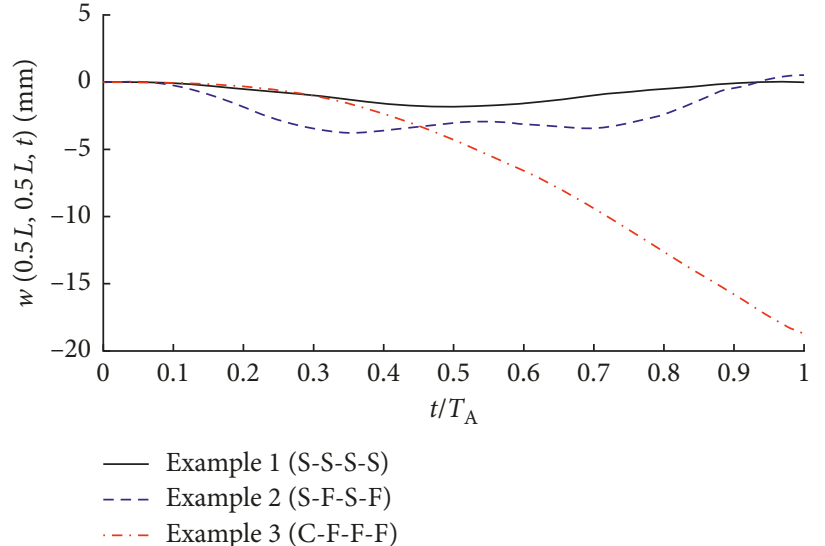

(a)

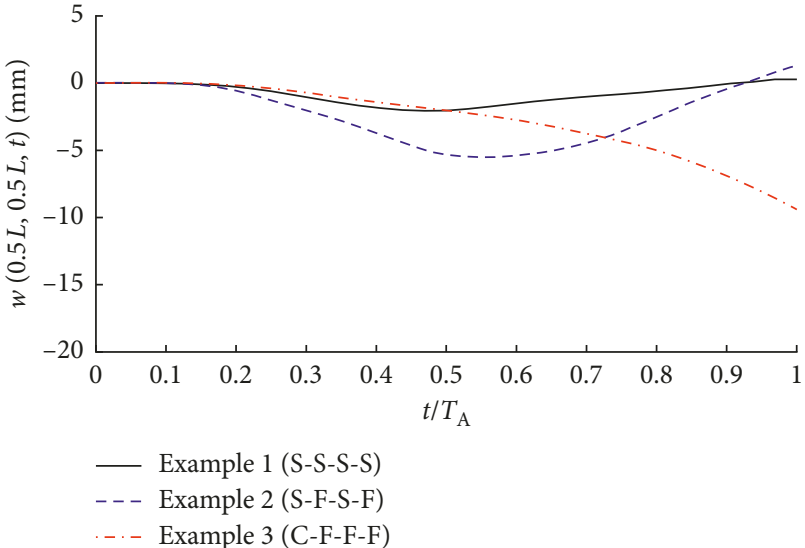

(b)

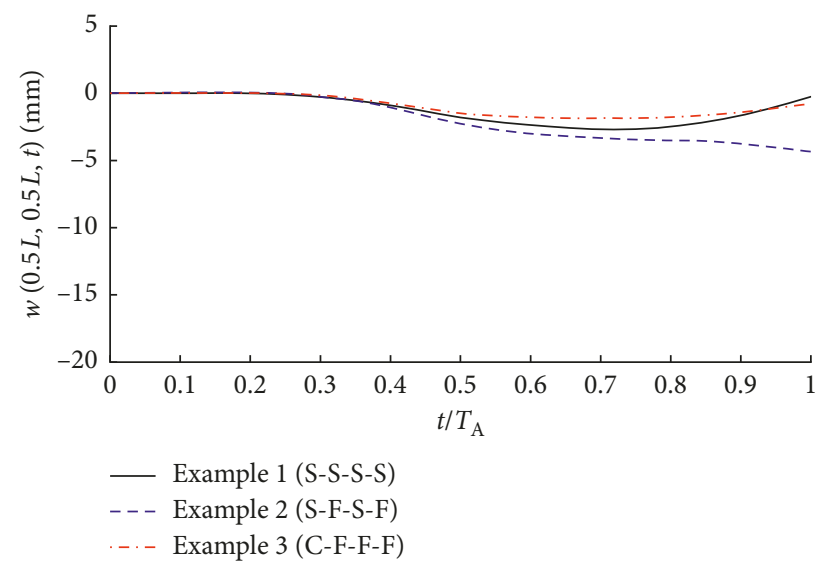

(c)

Figure 17: Effects of boundary conditions and moving speed $v_{0}$ on the vibration responses at the center of three square plates when a moving point force travels along the trajectory given by Case 2 of (39). (a) $v_{0}=1 \mathrm{~m} / \mathrm{s}$, (b) $v_{0}=2 \mathrm{~m} / \mathrm{s}$, and (c) $v_{0}=5 \mathrm{~m} / \mathrm{s}$.

Figure 20 shows the effects of boundary conditions on the vibration responses at the center of the first span $(X=Y=0.5 L)$, second $\operatorname{span}(X=2 L, Y=0.5 L)$, and third span $(X=3.5 L, Y=0.5 L)$ of the S-S-S-S (Example 4) and C-S-S-S
(Example 5) supports of three-span rectangular plates subjected to the moving point force whose trajectory is given by (40). The vibration amplitudes of the S-S-S-S supports (Example 4) are found to be larger than those of the C-S-S-S 


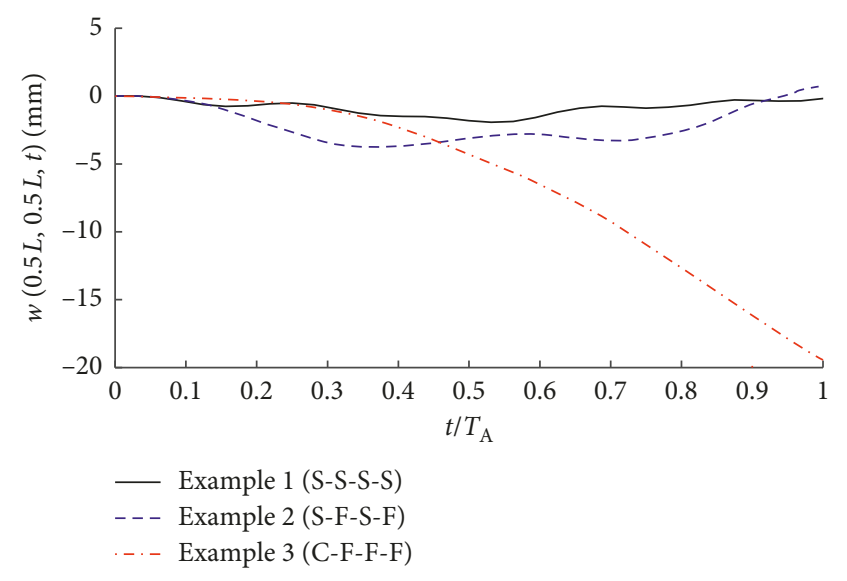

(a)

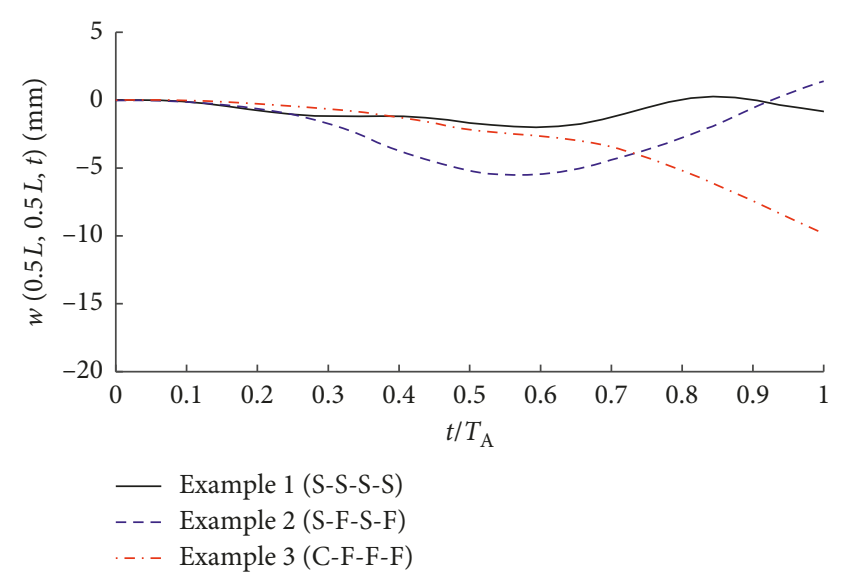

(b)

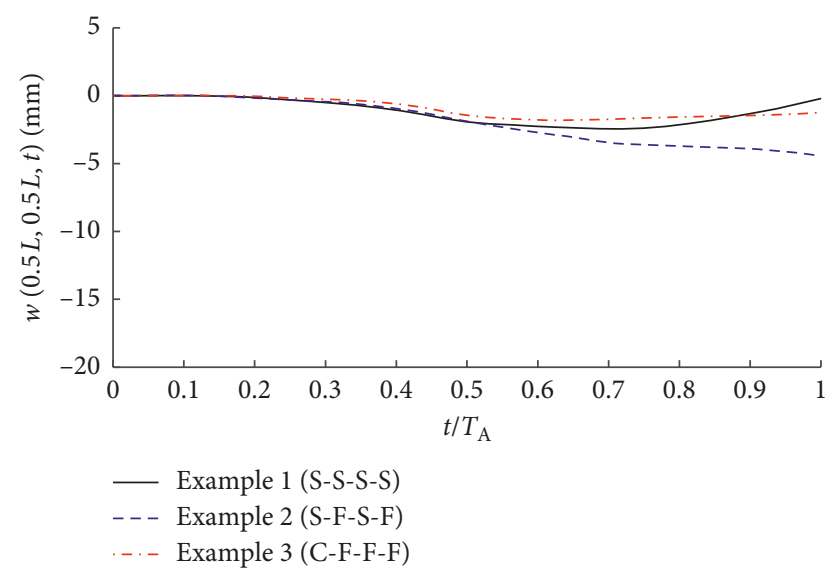

(c)

Figure 18: Effects of boundary conditions and moving speed $v_{0}$ on the vibration responses at the center of three square plates when a moving point force travels along the trajectory given by Case 3 of (39). (a) $v_{0}=1 \mathrm{~m} / \mathrm{s}$, (b) $v_{0}=2 \mathrm{~m} / \mathrm{s}$, and (c) $v_{0}=5 \mathrm{~m} / \mathrm{s}$.

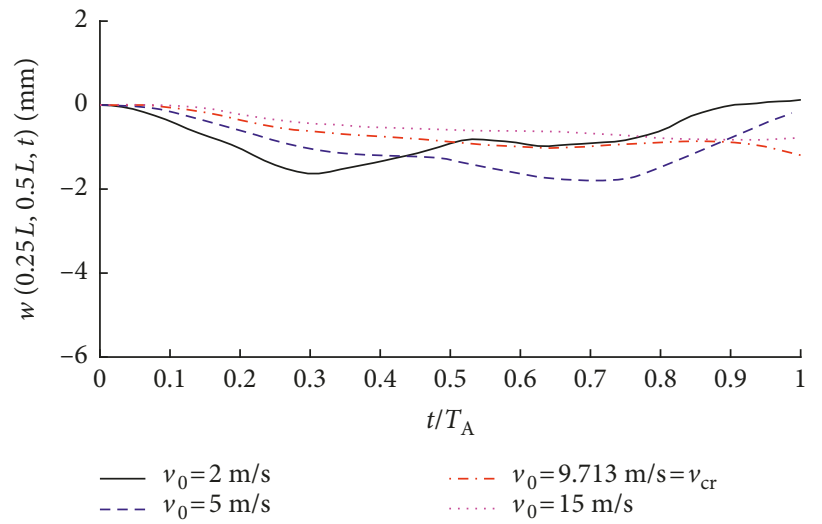

(a)

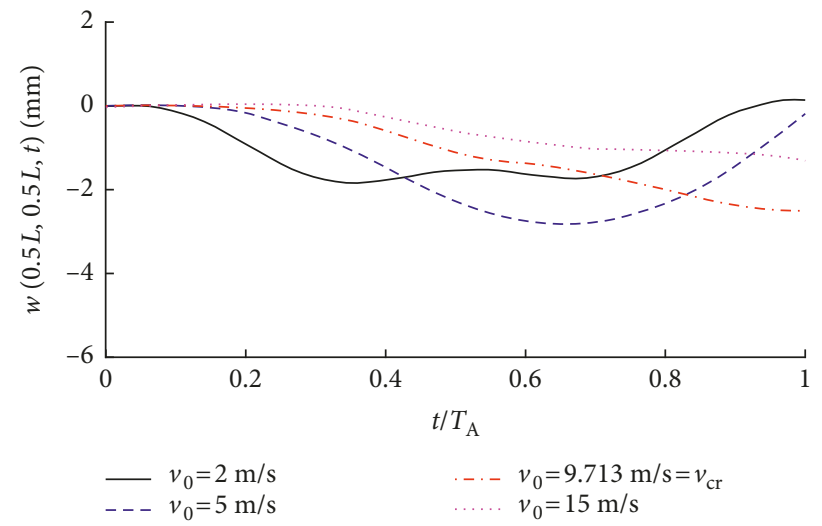

(b)

Figure 19: Continued. 


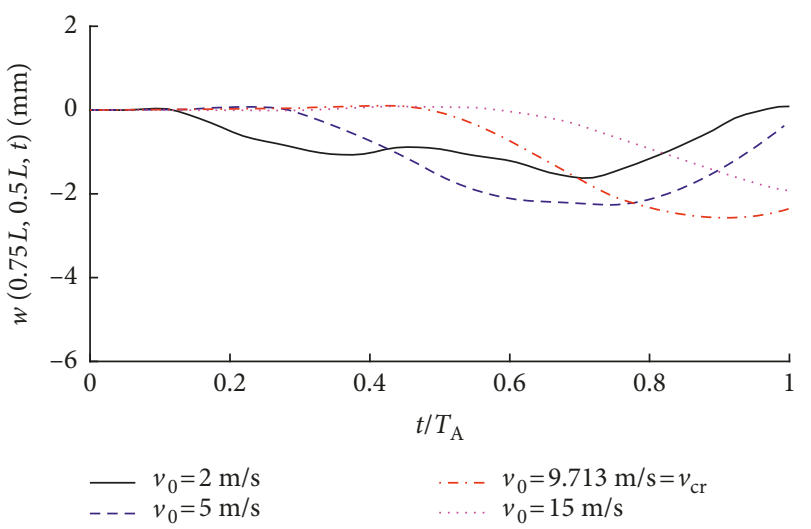

(c)

FIGURE 19: Effects of the moving speed $v_{0}$ on the vibration response of the S-S-S-S square plate (Example 1) subjected to a moving point force whose trajectory is given by Case 1 of (39), where $v_{\text {cr }}$ is the critical speed [5]. (a) At $x=0.25 L, y=0.5 L$, (b) at $x=0.5 L, y=0.5 L$, and (c) at $x=0.75 L, y=0.5 L$.

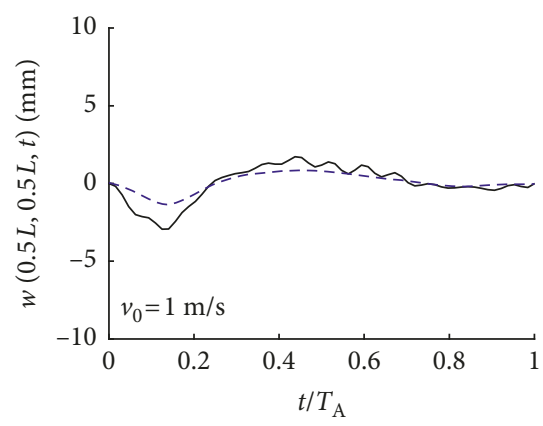

Example 4 (S-S-S-S)

- - - Example 5 (C-S-S-S)

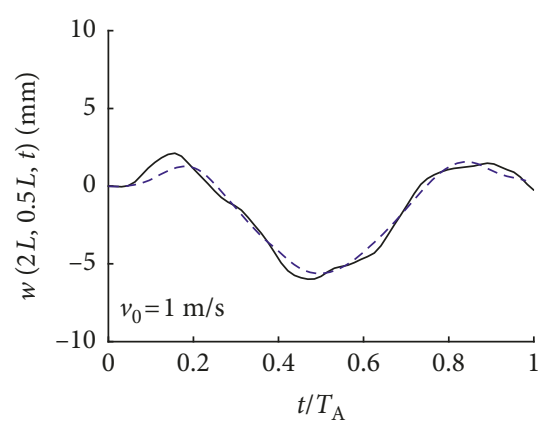

— Example 4 (S-S-S-S)

- - Example 5 (C-S-S-S)
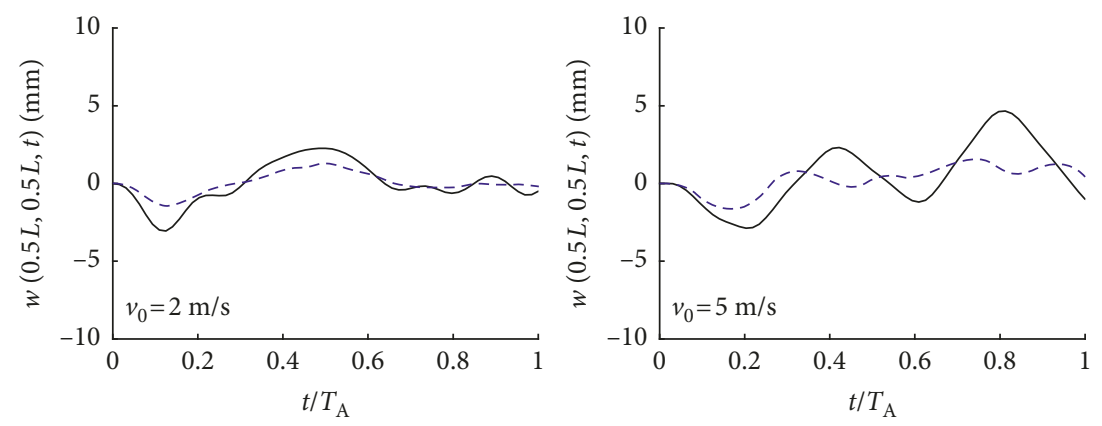

(a)
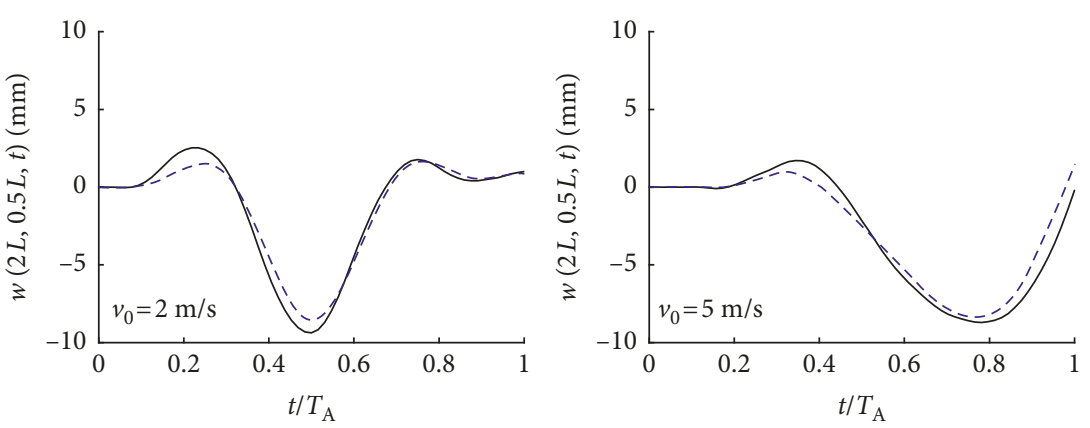

(b)

Figure 20: Continued. 

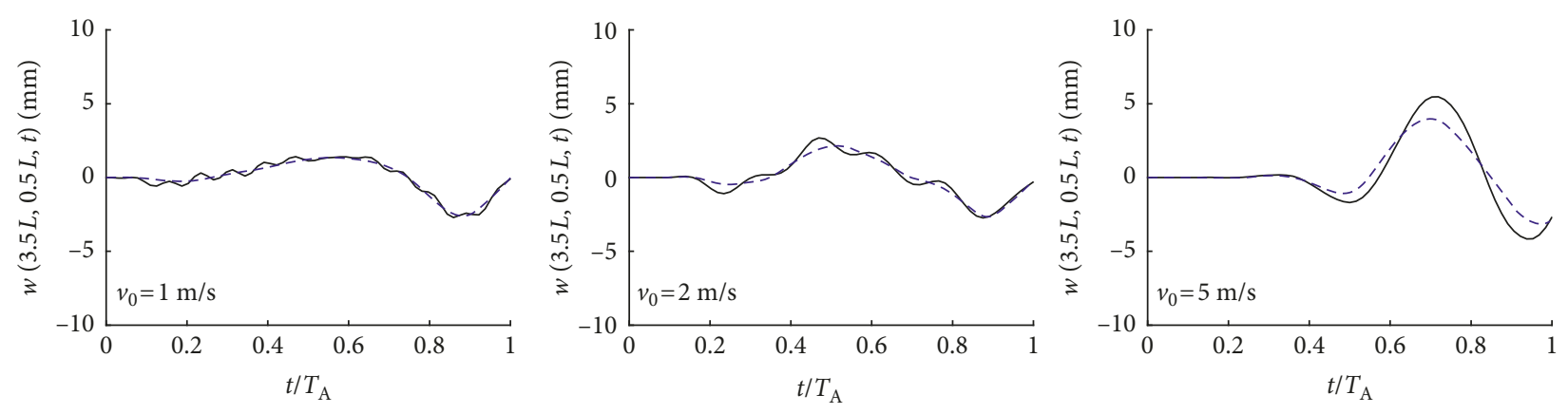

- Example 4 (S-S-S-S)

- - - Example 5 (C-S-S-S)

(c)

FIGURE 20: Effects of the boundary conditions on the vibration responses of two three-span rectangular plates subjected to a moving point force whose trajectory is given by (40). (a) At $X=0.5 L, Y=0.5 \mathrm{~L}$, (b) at $X=2 \mathrm{~L}, Y=0.5 \mathrm{~L}$, and (c) at $X=3.5 \mathrm{~L}, Y=0.5 \mathrm{~L}$.
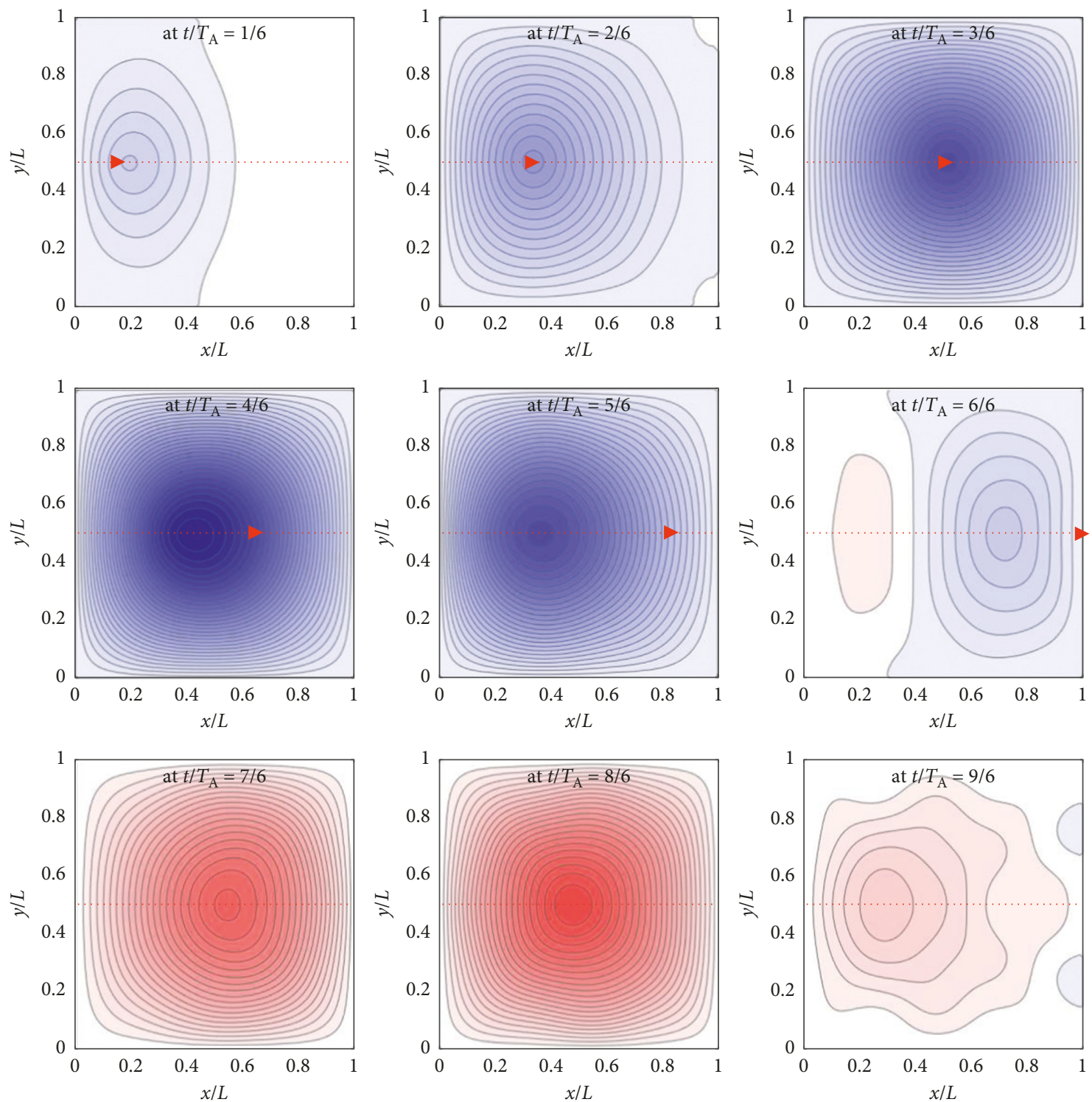

(a)

Figure 21: Continued. 

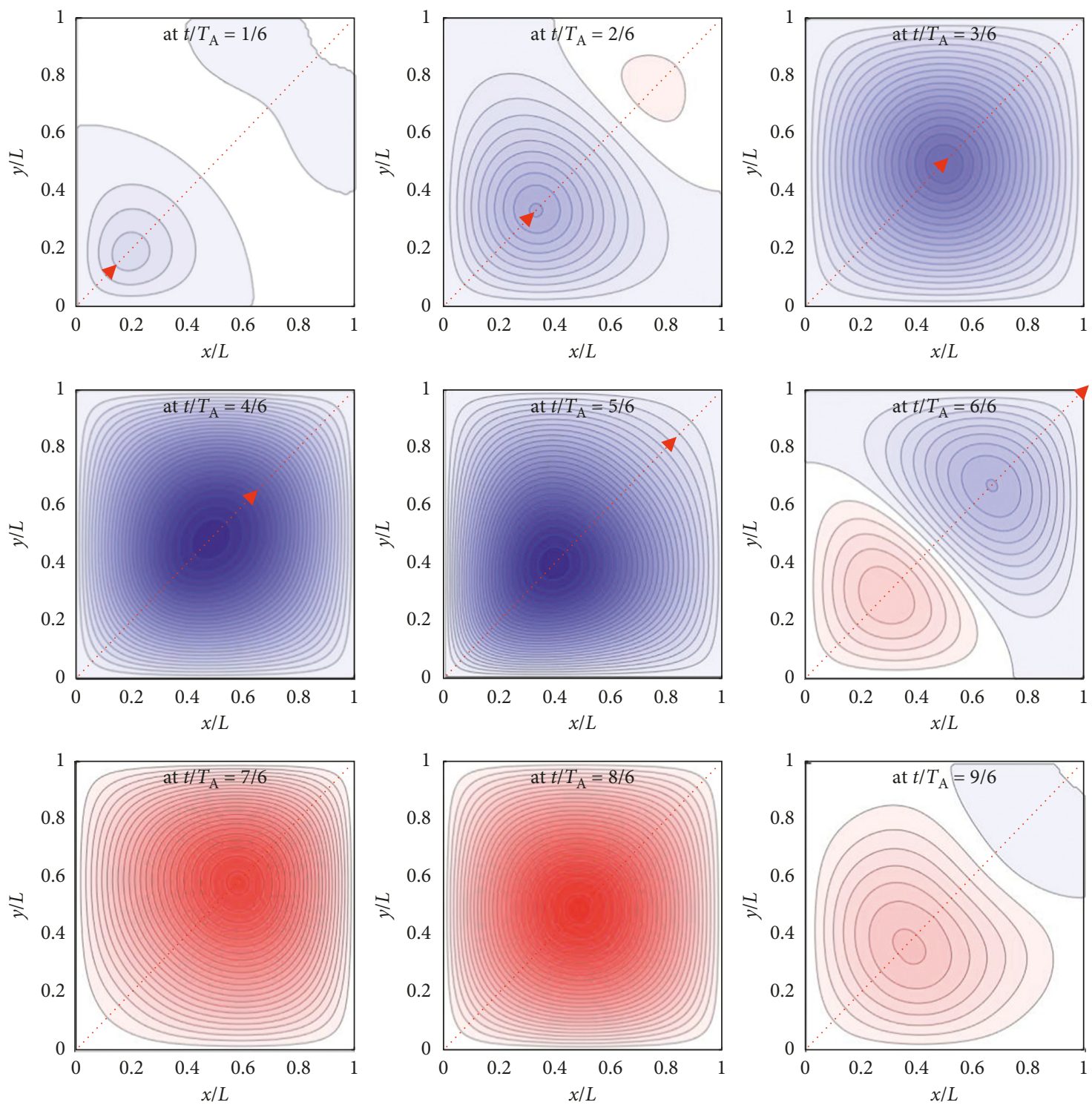

(b)

Figure 21: Continued. 

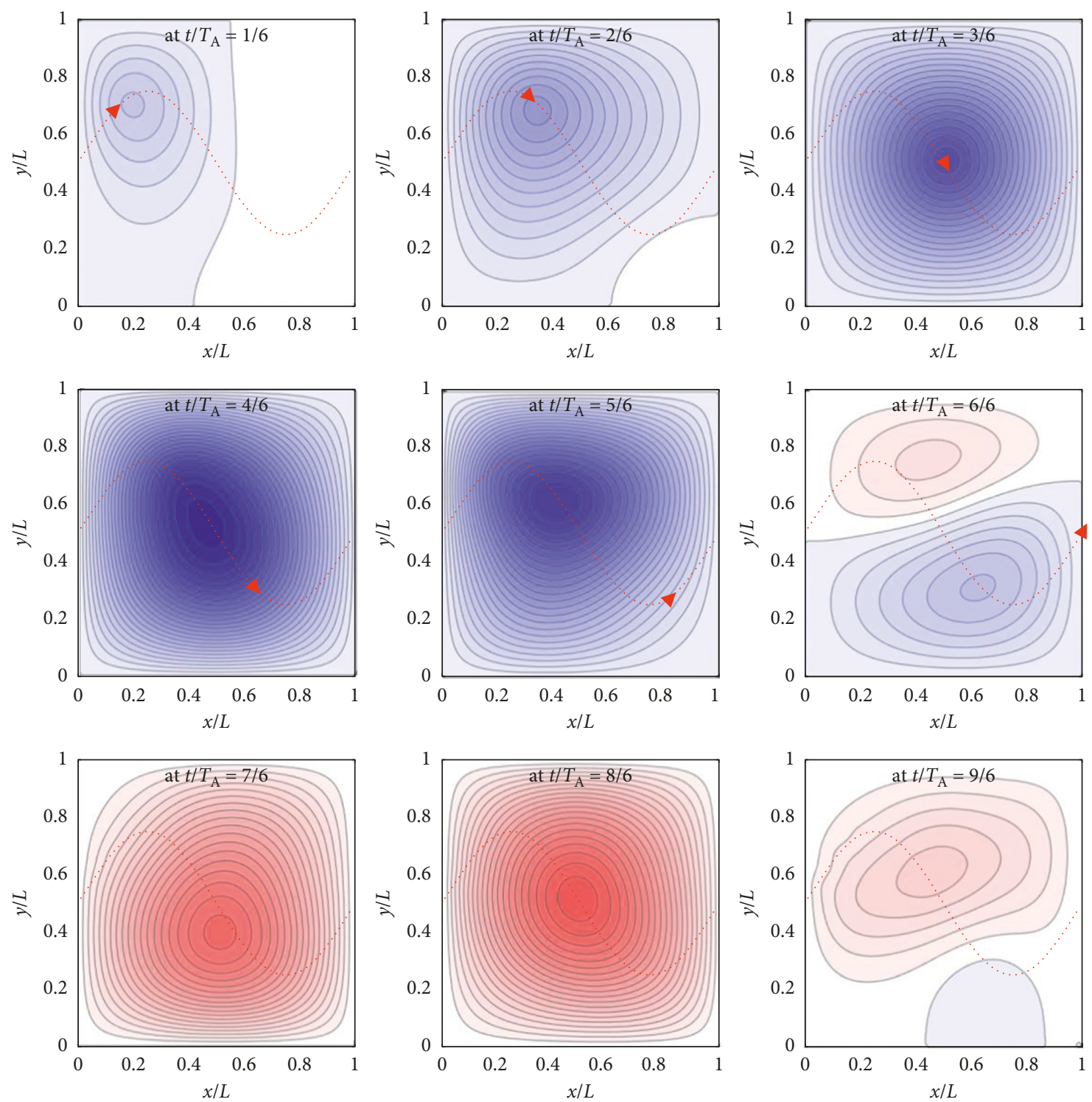

(c)

FIGURE 21: (a) Effects of the trajectory of a moving point force on the deformed shape of the S-S-S-S square plate (Example 1) when the moving point force travels at $v_{0}=5 \mathrm{~m} / \mathrm{s}$ along the trajectory given by Case 1 of (39), where the red arrow indicates current position of the moving point force. (b) Effects of the trajectory of a moving point force on the deformed shape of the S-S-S-S square plate (Example 1) when the moving point force travels at $v_{0}=5 \mathrm{~m} / \mathrm{s}$ along the trajectory given by Case 2 of (39), where the red arrow indicates current position of the moving point force. (c) Effects of the trajectory of a moving point force on the deformed shape of the S-S-S-S square plate (Example 1) when the moving point force travels at $v_{0}=5 \mathrm{~m} / \mathrm{s}$ along the trajectory given by Case 3 of (39), where the red arrow indicates current position of the moving point force.

supports (Example 5), at all the locations and moving speeds considered. In general, the vibration amplitudes at the center of the first and third spans tend to increase as the moving speed $v_{0}$ increases.

Above observations confirm that the boundary conditions and moving speed of a moving point force are essential factors that determine the vibration responses of a multispan plate subjected to a moving point force. Especially, for a plate with preset boundary conditions, quite different dynamic behaviors are observed depending on whether the moving speed is larger than the critical speed or not.
5.3. Effects of the Trajectory of a Moving Point Force on the Vibration Responses. The present SEM is developed to consider any types of moving force trajectories. Thus, we considered square plates with three different boundary conditions (Examples 1-3 in Figure 9) to investigate the effects of the trajectories of a moving point force on the deformed shapes of the example plates with time when the moving point force travels at the speed of $v_{0}=5 \mathrm{~m} / \mathrm{s}$. Three types of trajectories are considered: Case 1, Case 2, and Case 3 defined by (39).

Figures 21-23 show the effects of the trajectory of a moving point force on the deformed shapes of the S-S-S-S 

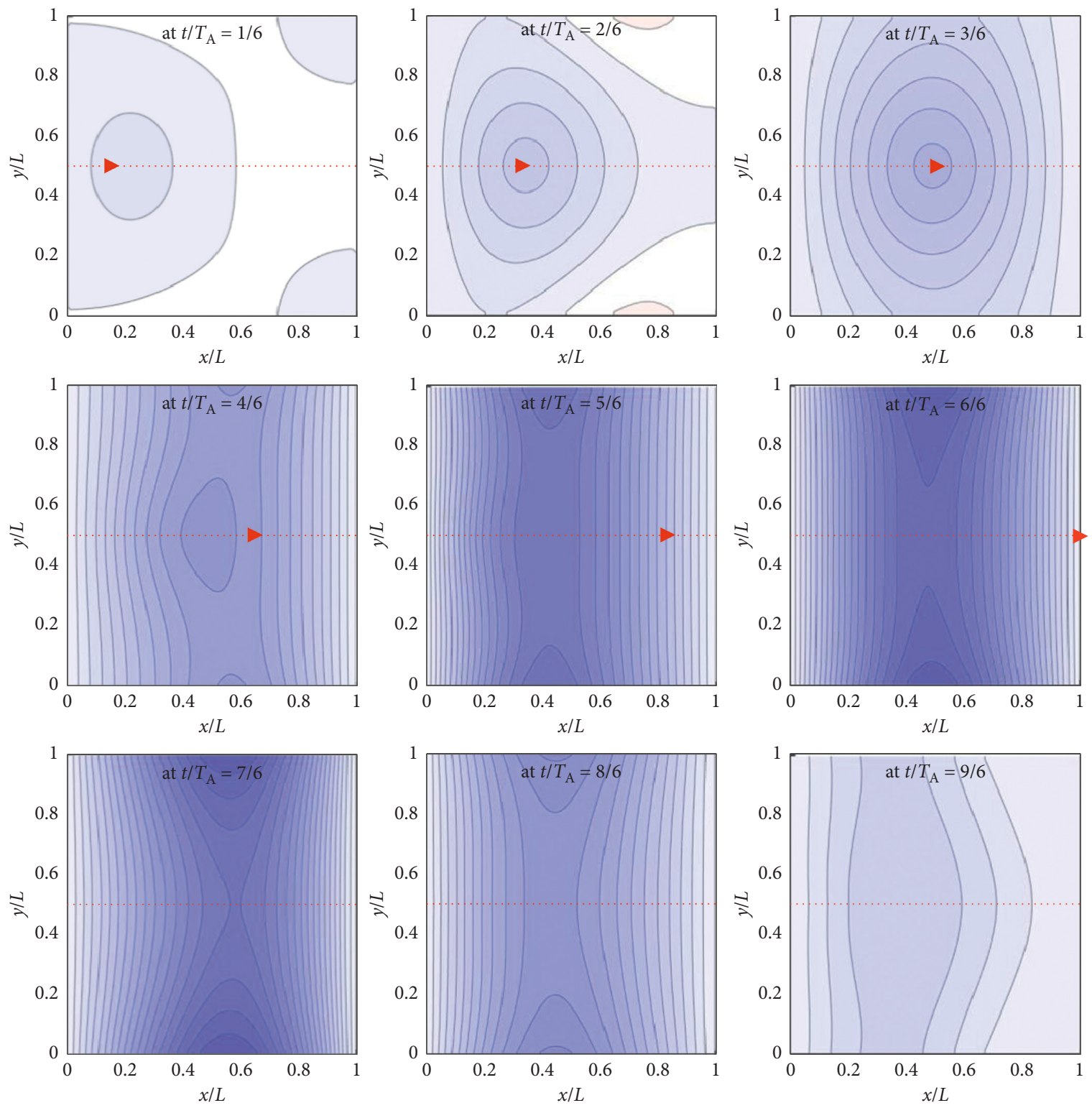

(a)

FIgUre 22: Continued. 

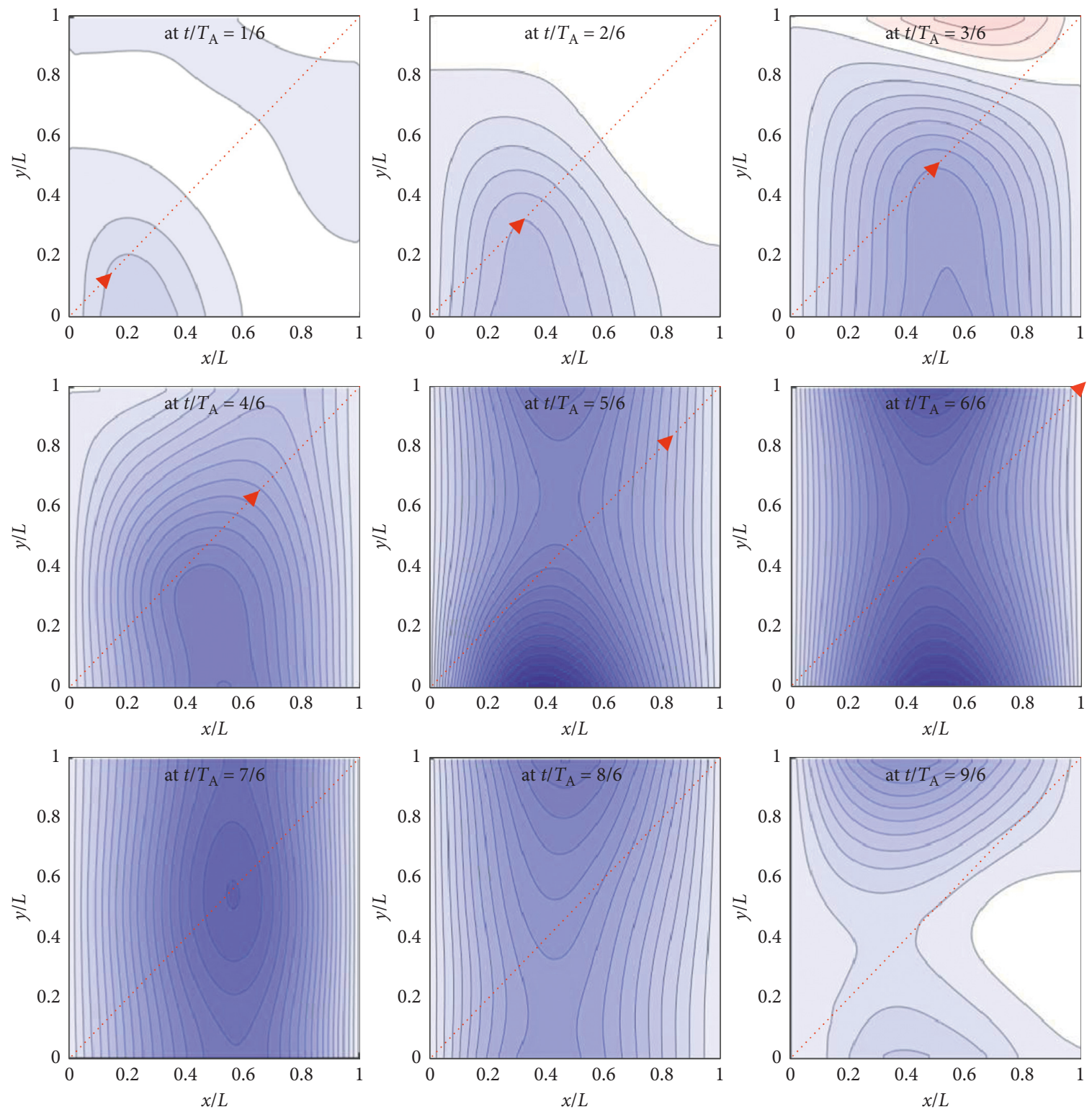

(b)

Figure 22: Continued. 

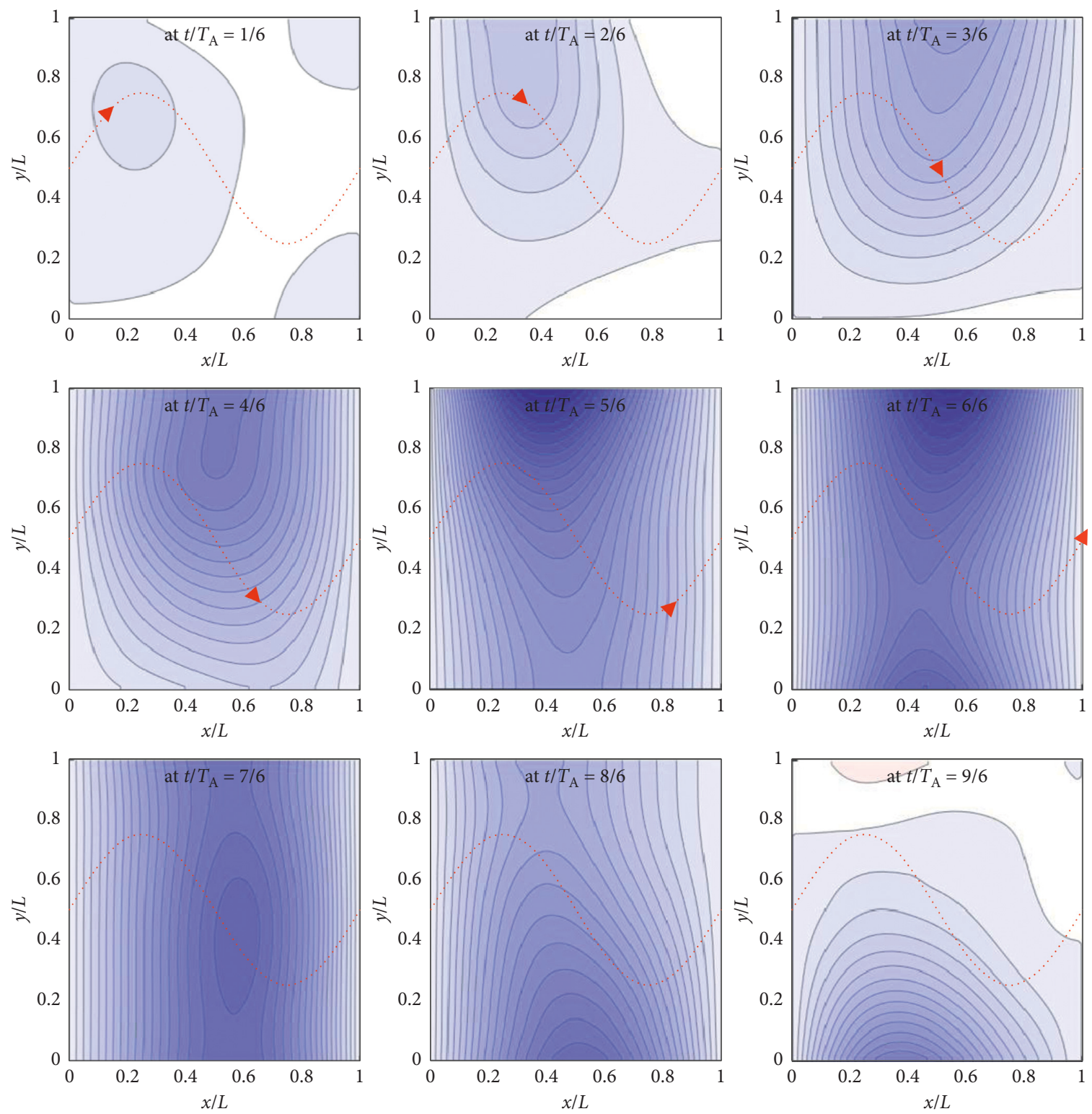

(c)

Figure 22: (a) Effects of the trajectory of a moving point force on the deformed shape of the S-F-S-F square plate (Example 2) when the moving point force travels at $v_{0}=5 \mathrm{~m} / \mathrm{s}$ along the trajectory given by Case 1 of (39), where the red arrow indicates current position of the moving point force. (b) Effects of the trajectory of a moving point force on the deformed shape of the S-F-S-F square plate (Example 2) when the moving point force travels at $v_{0}=5 \mathrm{~m} / \mathrm{s}$ along the trajectory given by Case 2 of (39), where the red arrow indicates current position of the moving point force. (c) Effects of the trajectory of a moving point force on the deformed shape of the S-F-S-F square plate (Example 2) when the moving point force travels at $v_{0}=5 \mathrm{~m} / \mathrm{s}$ along the trajectory given by Case 3 of (39), where the red arrow indicates current position of the moving point force.

(Example 1), S-F-S-F (Example 2), and C-F-F-F (Example 3) square plates. In Figures 21-23, the dotted lines denote the trajectories of a moving point force and the triangle marks denote the current locations and directions of the moving point force. The deformed shapes at nine dimensionless times $\left(t / T_{\mathrm{A}}=1 / 6,2 / 6,3 / 6, \ldots, 9 / 6\right.$, where $\left.T_{\mathrm{A}}=0.2 \mathrm{~s}\right)$ are represented by the contour lines, where the red-colored and blue-colored zones denote upward and downward deformations, respectively. From Figures 21-23, the following were found: (1) When $t / T_{\mathrm{A}}$ is small, the vibration amplitudes in general seem to have maximum values near the current position of the moving point force. However, when $t / T_{\mathrm{A}}$ becomes larger, the relationship between the location of the maximum vibration amplitude and the current position of the moving point force is not obvious. (2) Upward deformations can be observed at the free edges of Example 2 and Example 3. (3) As expected, the deformed shapes of Example 1, Example 2, and Example 3 are all symmetric with respect to the trajectory when the trajectory is given by Case 1 of (39). For the trajectory given by Case 2 of (39), only 

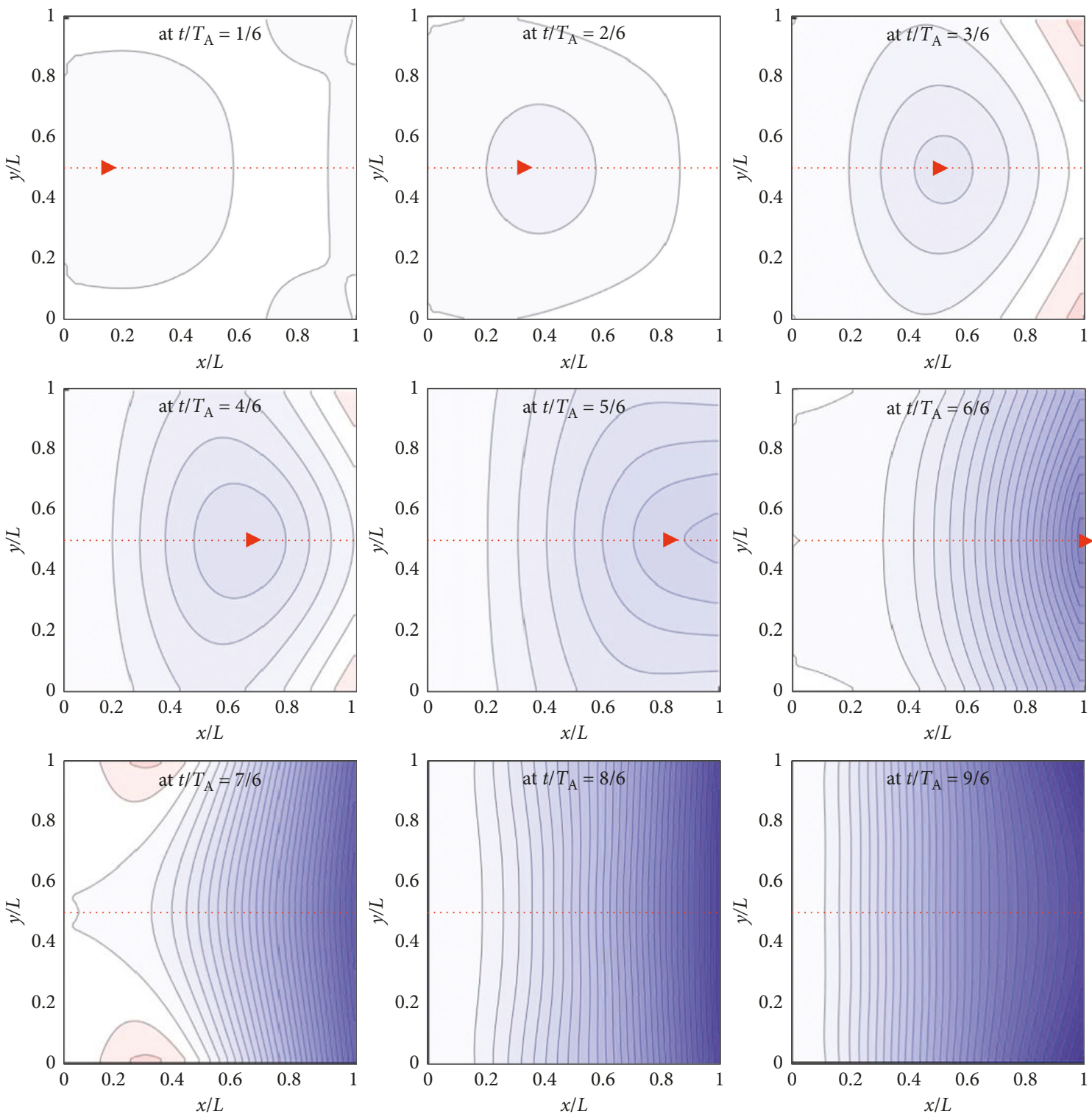

(a)

Figure 23: Continued. 

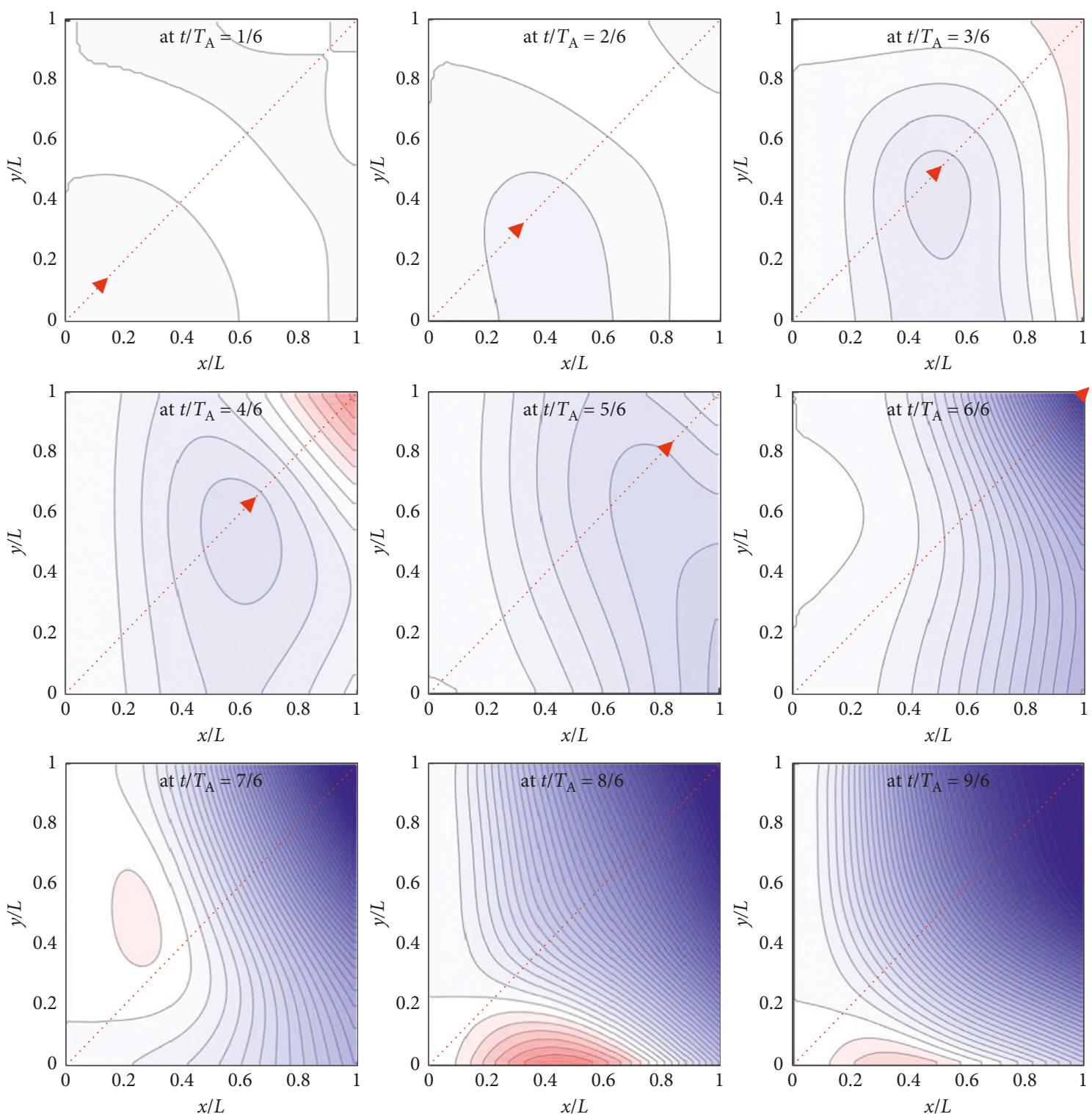

(b)

FIgure 23: Continued. 

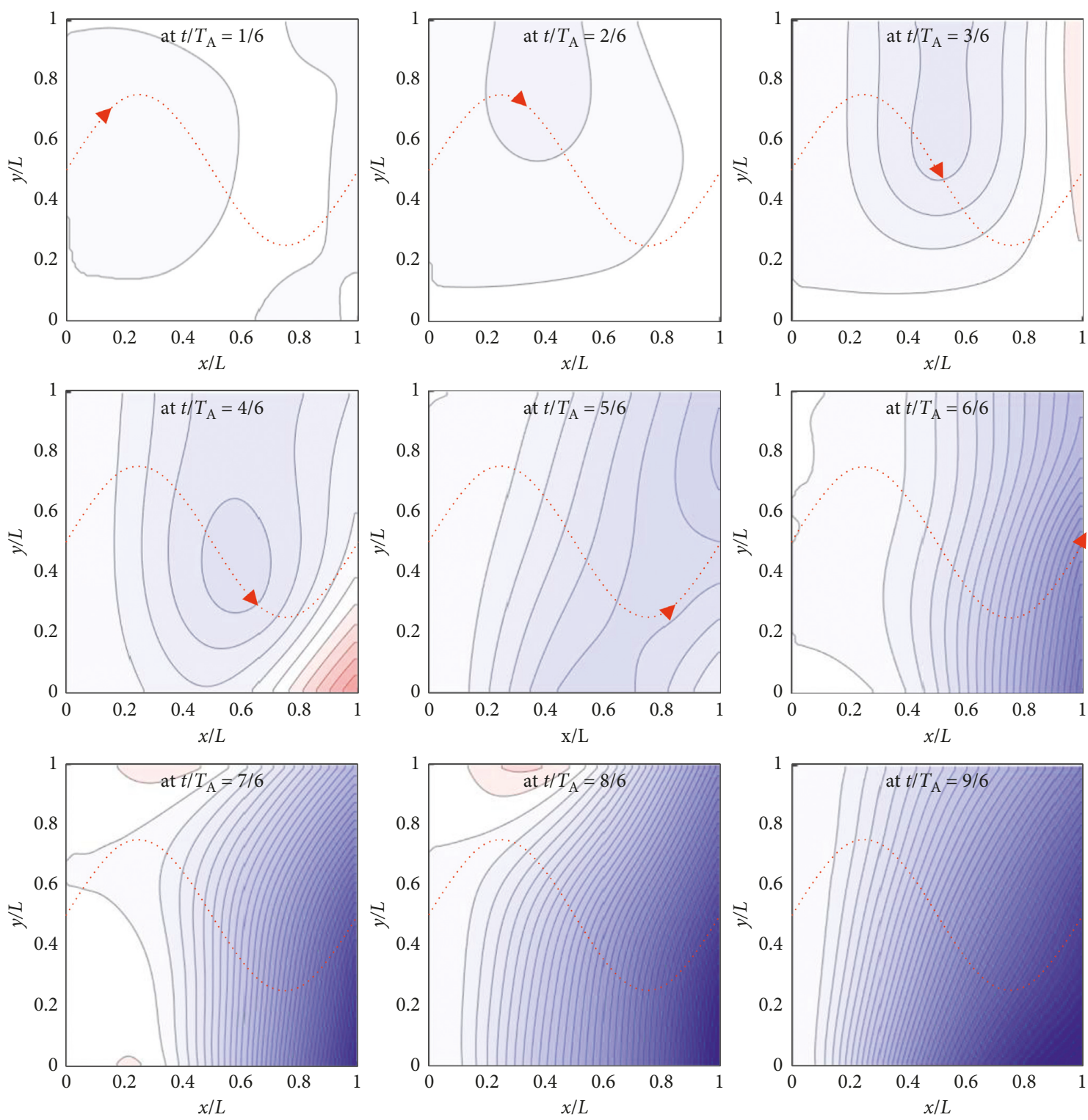

(c)

FIGURE 23: (a) Effects of the trajectory of a moving point force on the deformed shape of the C-F-F-F square plate (Example 3) when the moving point force travels at $v_{0}=5 \mathrm{~m} / \mathrm{s}$ along the trajectory given by Case 1 of (39), where the red arrow indicates current position of the moving point force. (b) Effects of the trajectory of a moving point force on the deformed shape of the C-F-F-F square plate (Example 3)) when the moving point force travels at $v_{0}=5 \mathrm{~m} / \mathrm{s}$ along the trajectory given by Case 2 of (39), where the red arrow indicates current position of the moving point force. (c) Effects of the trajectory of a moving point force on the deformed shape of the C-F-F-F square plate (Example 3) when the moving point force travels at $v_{0}=5 \mathrm{~m} / \mathrm{s}$ along the trajectory given by Case 3 of (39), where the red arrow indicates current position of the moving point force.

Example 1 shows such symmetry. However, for the trajectory given by Case 3 of (39), the symmetry of the deformed shapes cannot be observed for the example problems considered in this study.

In addition to the observations made in the previous section, when example plates are subjected to the same loading conditions such as the moving speed and trajectory, the effects of boundary conditions on the deformed shapes can be also clearly observed by comparing Figures 21(a), 22(a), and 23(a), Figures 21(b), 22(b), and 23(b), or Figures 21(c), $22(\mathrm{c})$, and $23(\mathrm{c})$.

\section{Conclusions}

In this study, a frequency-domain vibration analysis method was proposed for thin rectangular plates subjected to a moving point force, and it was applied to several single-span and multispan rectangular plates. The moving point force was transformed into the frequency domain as a series of stationary point forces distributed along the trajectory of the moving point force. Based on the spectral plate element model developed in the previous study [29], the plate span subjected to a stationary 
point force was represented by the four-element model, whereas all other plate spans, which were free from stationary point forces, were represented by the twoelement model. The time-domain vibration responses excited by the moving point force were obtained by using the superposition principle and inverse-FFT algorithm. The results are summarized as follows:

(1) The proposed frequency-domain representation of a moving point force can be applied to any type of moving point forces with arbitrary trajectories

(2) Based on the spectral plate element model developed in [29], the proposed SEM can be applied to any thin rectangular plates with arbitrary boundary conditions

(3) The high accuracy and computational efficiency of the proposed SEM are verified by comparison with the exact theory, ITM, FEM, and ANSYS

(4) Numerical results show that the time histories and deformed shapes of the single-span and multispan plates are dependent on the boundary conditions and on the speed and trajectory of the moving point force

(5) The proposed SEM can be readily applied to plates subjected to multiple moving point forces or moving distributed forces based on the superposition principle

The application of the spectral element model developed in this study will be limited to the thin plate structures represented by single or multiple rectangular finite plate elements. Thus, ongoing studies will be focused on the development of a spectral element model for the thick plate structures represented by triangular finite plate elements.

\section{Conflicts of Interest}

The authors declare that they have no conflicts of interest.

\section{Acknowledgments}

This work was supported by Inha University Research Grant (2018).

\section{References}

[1] T. H. T. Chan and D. B. Ashebo, "Theoretical study of moving force identification on continuous bridges," Journal of Sound and Vibration, vol. 295, no. 3-5, pp. 870-883, 2006.

[2] S. S. Law, J. Q. Bu, X. Q. Zhu, and S. L. Chan, "Moving load identification on a simply supported orthotropic plate," International Journal of Mechanical Science, vol. 49, no. 11, pp. 1262-1275, 2007.

[3] P. Śniady, "Dynamic response of a Timoshenko beam to a moving force," Journal of Applied Mechanics, vol. 75, no. 2, Article ID 024503, 4 pages, 2008.

[4] J. V. Amiri, A. Nikkhoo, M. R. Davoodi, and M. E. Hassanabadi, "Vibration analysis of a Mindlin elastic plate under a moving mass excitation by eigenfunction expansion method," ThinWalled Structures, vol. 62, pp. 53-64, 2013.

[5] L. Frýba, Vibration of Solids and Structures under Moving Loads, Noordhoff International Publishing, Groningen, Netherlands, 3rd edition, 1999.
[6] E. Ghafoori, M. H. Kargarnovin, and A. R. Ghahremani, "Dynamic responses of a rectangular plate under motion of an oscillator using a semi-analytical method," Journal of Vibration and Control, vol. 17, no. 9, pp. 1310-1324, 2011.

[7] C. I. Bajer and B. Dyniewicz, Numerical Analysis of Vibrations of Structures under Moving Inertial Load, Springer, Berlin, Germany, 2012.

[8] M. R. Shadnam, F. R. Rofooei, M. Mofid, and B. Mehri, "Periodicity in the response of nonlinear plate, under moving mass," Thin-Walled Structures, vol. 40, no. 3, pp. 283-295, 2002.

[9] Y. Yang, H. Ding, and L.-Q. Chen, "Dynamic response to a moving load of a Timoshenko beam resting on a nonlinear viscoelastic foundation," Acta Mechanica Sinica, vol. 29, no. 5, pp. 718-727, 2013.

[10] A. R. Vosoughi, P. Malekzadeh, and H. Razi, "Response of moderately thick laminated composite plates on elastic foundation subjected to moving load," Composite Structures, vol. 97, pp. 286-295, 2013.

[11] X. Wang and C. Jin, "Differential quadrature analysis of moving load problems," Advances in Applied Mathematics and Mechanics, vol. 8, no. 4, pp. 536-555, 2016.

[12] E. Esmailzadeh and M. Ghorashi, "Vibration analysis of Timoshenko beams subjected to a traveling mass," Journal of Sound and Vibration, vol. 199, no. 4, pp. 615-628, 1997.

[13] J. A. Gbadeyan and M. S. Dada, "Dynamic response of a Mindlin elastic rectangular plate under a distributed moving mass," International Journal of Mechanical Sciences, vol. 48, no. 3, pp. 323-340, 2006.

[14] J. S. Wu, M. L. Lee, and T. S. Lai, "The dynamic analysis of a flat plate under a moving load by the finite element method," International Journal for Numerical Methods in Engineering, vol. 24, no. 4, pp. 743-762, 1987.

[15] J. J. Wu, A. R. Whittaker, and M. P. Cartmell, "The use of finite element techniques for calculating the dynamic response of structures to moving loads," Computers \& Structures, vol. 78, no. 6, pp. 789-799, 2000.

[16] E. Ghafoori and M. Asghari, "Dynamic analysis of laminated composite plates traversed by a moving mass based on a firstorder theory," Composite Structures, vol. 92, no. 8, pp. 1865-1876, 2010.

[17] S. R. Mohebpour, P. Malekzadeh, and A. A. Ahmadzadeh, "Dynamic analysis of laminated composite plates subjected to a moving oscillator by FEM," Composite Structures, vol. 93, no. 6, pp. 1574-1583, 2011.

[18] P. Malekzadeh and S. M. Monajjemzadeh, "Nonlinear response of functionally graded plates under moving load," Thin-Walled Structures, vol. 96, pp. 120-129, 2015.

[19] K. Henchi, M. Fafard, G. Dhatt, and M. Talbot, "Dynamic behaviour of multi-span beams under moving loads," Journal of Sound and Vibration, vol. 199, no. 1, pp. 33-50, 1997.

[20] Y. A. Dugush and M. Eisenberger, "Vibrations of nonuniform continuous beams under moving loads," Journal of Sound and Vibration, vol. 254, no. 5, pp. 911-926, 2002.

[21] N. Azizi, M. M. Saadatpour, and M. Mahzoon, "Using spectral element method for analyzing continuous beams and bridges subjected to a moving load," Applied Mathematical Modelling, vol. 36 , no. 8, pp. 3580-3592, 2012.

[22] V. Sarvestan, H. R. Mirdamadi, M. Ghayour, and A. Mokhtari, "Spectral finite element for vibration analysis of cracked viscoelastic Euler-Bernoulli beam subjected to moving load," Acta Mechanica, vol. 226, no. 12, pp. 42594280, 2015. 
[23] Y. Song, T. Kim, and U. Lee, "Vibration of a beam subjected to a moving force: frequency-domain spectral element modeling and analysis," International Journal of Mechanical Sciences, vol. 113, pp. 162-174, 2016.

[24] T. Kim and U. Lee, "Dynamic analysis of a multi-span beam subjected to a moving force using the frequency domain spectral element method," Computers and Structures, vol. 192, pp. 181-195, 2017.

[25] F. Shirmohammadi, S. Bahrami, M. M. Saadatpour, and A. Esmaeily, "Modeling wave propagation in moderately thick rectangular plates using the spectral element method," Applied Mathematical Modelling, vol. 39, no. 12, pp. 3481-3495, 2015.

[26] J. F. Doyle, Wave Propagation in Structures: Spectral Analysis using Fast Discrete Fourier Transforms, Springer, New York, NY, USA, 1997.

[27] U. Lee, Spectral Element Method in Structural Dynamics, John Wiley \& Sons, Singapore, 2009.

[28] T. Kim and U. Lee, "Modified one-element method for exact dynamic responses of a beam by using the frequency domain spectral element method," International Journal of Mechanical Sciences, vol. 119, pp. 333-342, 2016.

[29] I. Park, T. Kim, and U. Lee, "Frequency domain spectral element model for the vibration analysis of a thin plate with arbitrary boundary conditions," Mathematical Problems in Engineering, vol. 2016, Article ID 9475397, 20 pages, 2016.

[30] I. Park and U. Lee, "A generic type of frequency-domain spectral element model for the dynamics of a laminated composite plate," Composite Structures, vol. 172, no. 15, pp. 83-101, 2017.

[31] W. C. Reynolds, Solution of Partial Differential Equations, Department of Mechanical Engineering, Stanford University, CA, USA, 1981.

[32] F. Birgersson, S. Finnveden, and C.-M. Nilsson, "A spectral super element for modelling of plate vibration. Part 1: general theory," Journal of Sound and Vibration, vol. 287, no. 1-2, pp. 297-314, 2005.

[33] L. Meirovitch, Principles and Techniques of Vibrations, Prentice-Hall, NJ, USA, 1997.

[34] E. Kreyszig, Advanced Engineering Mathematics, John Wiley \& Sons, New York, NY, USA, 1972.

[35] D. E. Newland, Random Vibrations: Spectral and Wavelet Analysis, Longman, New York, NY, USA, 3rd edition, 1993.

[36] D. E. Dudgeonand and R. M. Mersereau, Multidimensional Digital Signal Processing, Prentice-Hall, NJ, USA, 1984.

[37] R. D. Blevins, Formulas for Natural Frequency and Mode Shape, Van Nostrand Reinhold Company, New York, NY, USA, 1979.

[38] M. Petyt, Introduction to Finite Element Vibration Analysis, Cambridge University Press, New York, NY, USA, 2010.

[39] ANSYS Release 11.0 Documentation for ANSYS, ANSYS, Inc., Canonsburg, PA, USA, 2006.

[40] MATLAB User's Guide, The MathWorks, Natick, MA, USA, 1993. 


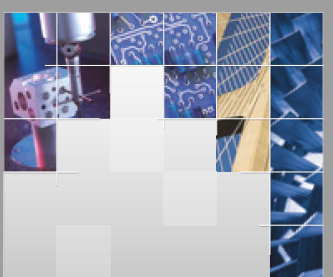

\section{Enfincering}
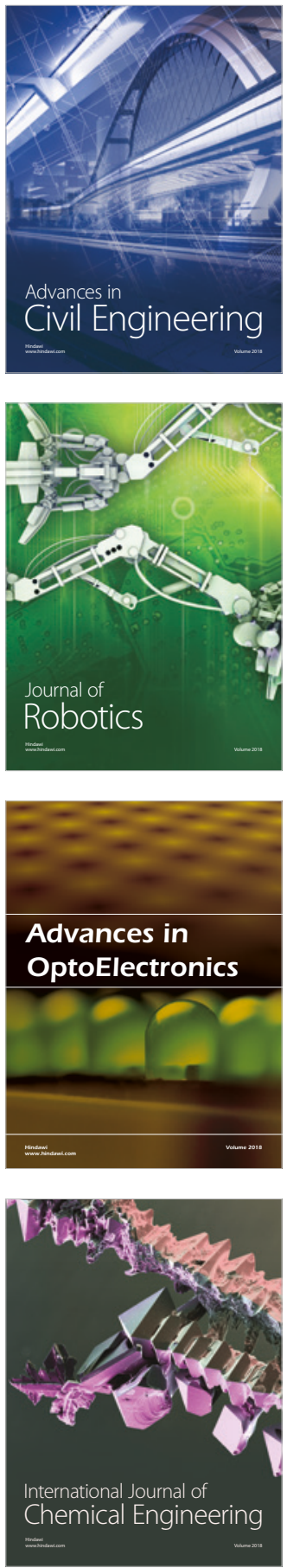

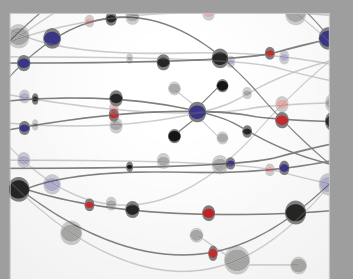

\section{Rotating \\ Machinery}

The Scientific World Journal

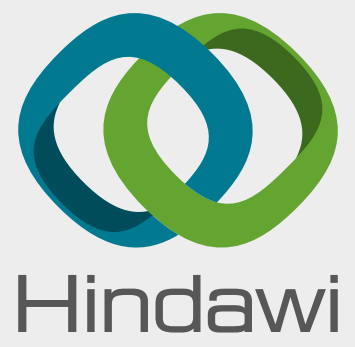

Submit your manuscripts at

www.hindawi.com
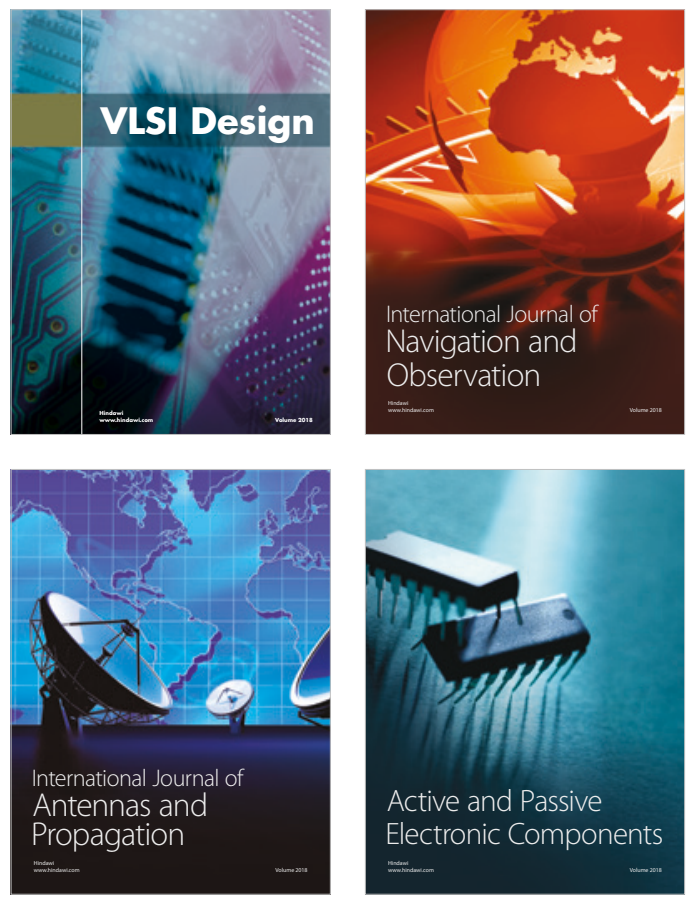
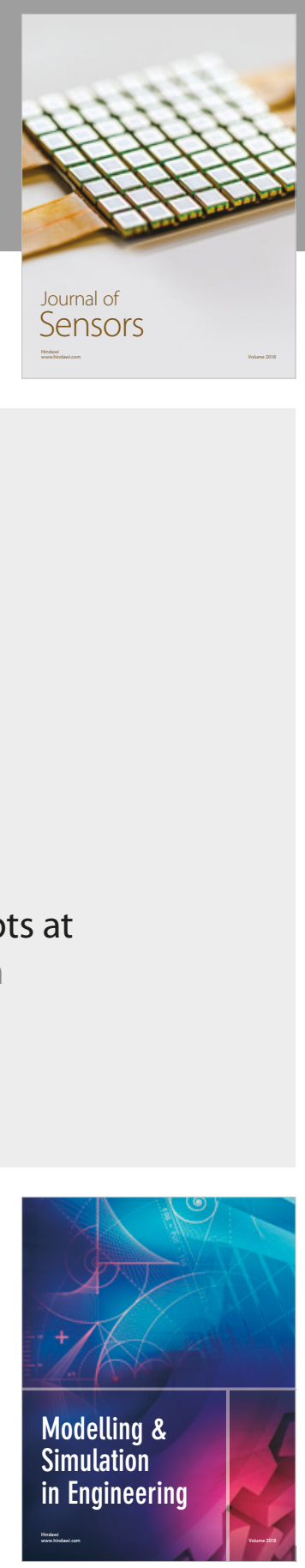

\section{Advances \\ Multimedia}
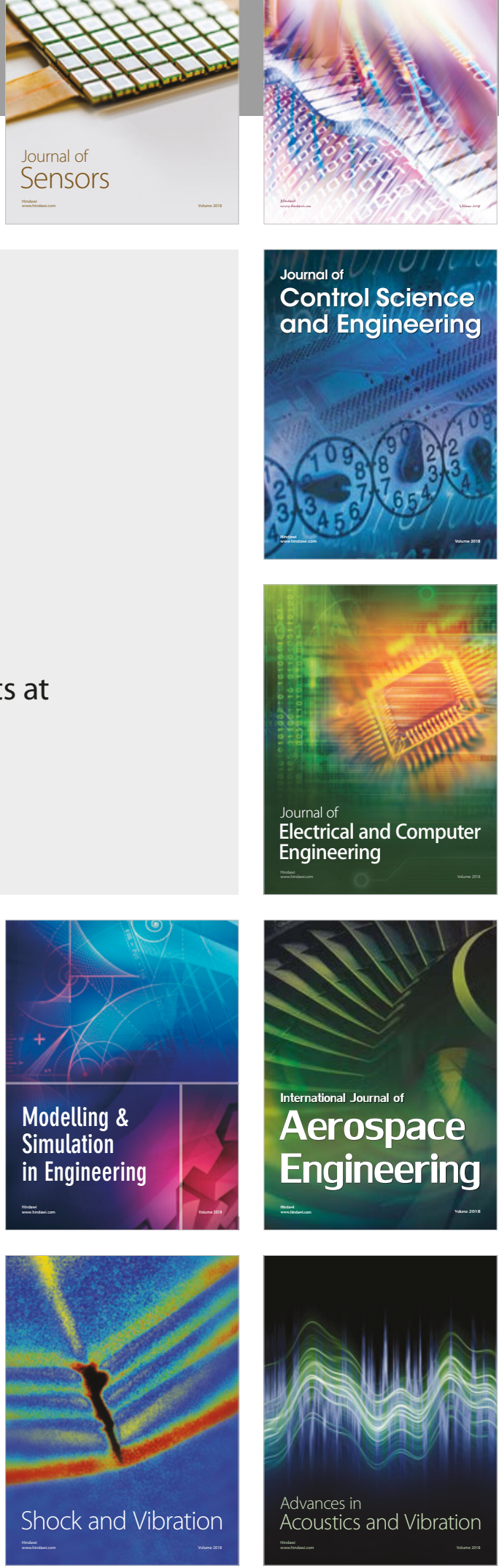